\title{
Application of an Improved DCGAN for Image GenerationApplication of an Improved DCGAN for Image Generation
}

\section{Bingqi Liu}

Chengdu University of Technology

Jiwei Lv ( $\boldsymbol{\nabla}$ lvjiwei_cd@163.com )

Chengdu University of Technology https://orcid.org/0000-0003-2310-725X

\section{Xinyue Fan}

Chengdu University of Technology

Jie Luo

Chengdu University of Technology

\section{Tianyi Zou}

Chengdu University of Technology

\section{Research Article}

Keywords: computer vision, DCGAN, deep learning, GANs, image generation

Posted Date: May 3rd, 2021

DOI: https://doi.org/10.21203/rs.3.rs-266104/v1

License: (c) (1) This work is licensed under a Creative Commons Attribution 4.0 International License. Read Full License 


\title{
Application of an Improved DCGAN for Image Generation
}

\author{
Bingqi Liu', ${ }^{1,2}$ Jiwei Lv², Xinyue Fan², Jie Luo², and Tianyi Zou \\ ${ }^{1}$ School of Mechanical Engineering, Chengdu University, Chengdu, 610106, China \\ ${ }^{2}$ Geomathematics Key Laboratory of Sichuan Province, Chengdu University of Technology, Chengdu, 610059, China \\ Corresponding author: Jiwei Lv (e-mail: 1vjiwei_cd@163.com)
}

\begin{abstract}
With the rapid development of deep learning, image generation technology has become one of the current hot research areas. A deep convolutional generative adversarial network (DCGAN) can better adapt to complex image distributions than other methods. In this paper, based on a traditional generative adversarial networks (GANs) image generation model, first, the fully connected layer of the DCGAN is further improved. To solve the problem of gradient disappearance in GANs, the activation functions of all layers of the discriminator are LeakyReLU functions, the output layer of the generator uses the Tanh activation function, and the other layers use ReLU. Second, the improved DCGAN model is verified on the MNIST dataset, and simple initial fraction (ISs) and complex initial fraction (ISc) indexes are established from the two aspects of image quality and image generation diversity, respectively. Finally, through a comparison of the two groups of experiments, it is found that the quality of images generated by the DCGAN model constructed in this paper is 2.02 higher than that of the GANs model, and the diversity of the images generated by the DCGAN is 1.55 higher than that of GANs. The results show that the improved DCGAN model can solve the problem of low-quality images being generated by the GANs and achieve good results.
\end{abstract}

INDEX TERMS computer vision, DCGAN, deep learning, GANs, image generation

\section{INTRODUCTION}

With the introduction of the concepts of cloud computing and big data and the rapid development of computer hardware facilities, deep learning has undergone rapid development and has been used in many applications in recent years. However, the development of image generation technology is slow in several branches of deep learning. Before GANs were proposed, the main image generators were automatic regression models [1] and variational autoencoders [2]. At the same time, based on the improvements in GANs theory, GANs has been applied in image conversion, image feature extraction and other fields.

As a new type of image generation model, GANs have attracted the attention of many researchers, who have gradually improved and provided a large number of mature image generation frameworks (such as DCGAN, CGAN, Pix2Pix, etc.). In terms of theoretical research on GANs, in 2014, Goodfellow et al. first described a new image generation model, the GANs, which is composed of a generator and a discriminator [3]. In the same year, Mehdi et al. were inspired by the introduction of convolutional neural networks on the basis of GANs and proposed the CGAN, which solved the unstable training behavior problem of GANs by adding category labels [4]. To solve the instability of GANs, in 2016, Goodfellow and Salimans et al. proposed stabilizing the training process of DCGAN with feature matching, small batch recognition and historical averaging, and this work provided a basis for follow-up research [5]. With regard to the applications of GANs, Isola et al. implemented image conversion using Pix2Pix and paired training data [6]; Zhang et al. proposed StackGAN, which first generates basic images and text descriptions based on the original image information, and then improved the process to generate high-resolution images [7]. In 2017, Zhu et al. proposed CycleGAN, which solved the problem of Pix2Pix needing paired data, and proposed the cycle-consistency loss function to realize image conversion from a horse to a zebra [8]. Karras proposed StyleGAN in 2018 to accelerate and stabilize the training speed of the network by gradually increasing the numbers of generators and discriminators [9]. This method uses natural style-conversion technology, such as adaptive instance normalization (AdaIN), for reference purposes and realizes the real-time transmission of any style [10]. BigGAN was proposed by Andrew Brock in 2019. BigGAN adopts a selfattention mechanism and spectral normalization, and it is a good model for image generation on ImageNet at present [11]. In summary, there have been some comprehensive 
methods proposed to solve the problems of GANs generation and resolution in recent years.

For the first time, Alec Radford et al. proposed DCGAN [12]. Liu et al compared the unconstrained DCGAN and the constrained DCGAN, and the results showed that after adding constraints during the training phase, the DCGAN model significantly improved upon the results of the virtual face generation model, thus demonstrating the enhanced ability of the generator and discriminator [13]. Mahmoud and Guo used the DCGAN to extract depth features for strongly representing TSR images [14]. Fang et al. proposed a new gesture recognition algorithm based on a convolutional neural network and the DCGAN and applied this method to expression recognition, calculation and text output, achieving good results in all cases [15]. The actual image and noise vector of the DCGAN were trained, and smoke image training was used to generate a discriminator, thereby showing that the DCGAN can effectively monitor smoke images [16]. The biggest differences between the DCGAN and original GANs are that the DCGAN uses a convolutional neural network $(\mathrm{CNN})$ to replace the multilayer perceptron in the original GANs, removes the pooling layer, and uses a convolution with a defined step size to replace the upper sampling layer for improving the stability of the training process.

The remainder of the paper is organized as follows: The Section I summarizes the progress of research with regard to GANs and the DCGAN; the Section II mainly introduces the principles of the improved DCGAN algorithm and designs the network structure; the Section III constructs the image generation models, with one based on GANs and the other based on the DCGAN; the Section IV introduces two image generation quality evaluation methods and analyzes the effects of the two models on image generation quality and image diversity. The study's discussion and conclusions are presented in Section V.

\section{IMPROVED DESIGN OF THE STRUCTURE OF THE} DCGAN

\section{A. PRINCIPLES OF THE DCGAN ALGORITHM}

The adversarial training process of the DCGAN model established in this paper was calculated by using (1):

$\min _{G} \max _{D} V(D, G)=\mathrm{E}_{x \sim} p_{d a t a^{(x)}} \log [D(x)]+\mathrm{E}_{Z \sim p_{Z^{(Z)}}}[\log (1-D(G(z)))]$

where $P_{\text {data }}$ is the distribution of the real data, $x$ is the sample image data, $P_{Z}$ represents arbitrarily distributed noise, $Z$ expresses the number of random vectors in $P_{Z}$, and $\mathrm{E}$ expresses expectations.

The first step is to find the minimum cross entropy of the discriminator $D$ under the condition where a generator $G$ is given. The objective function was calculated by using (2):

$$
\operatorname{Obj}(D)=-\mathrm{E}_{x \sim P_{\text {data }}} \log [D(x)]-\mathrm{E}_{Z \sim P_{Z}} \log [1-D(G(Z))]
$$

where $\log [D(x)]$ is used to judge the sample data, $\log [1-D(G(Z))]$ represents the judgment of the generated sample data, that is, the closeness of the distribution of the sample data output by the discriminator $P_{\text {data }}$ and the data distribution generated by $G P_{G}(x)$, and $x$ is a sample from the real data. According to (3):

$$
\begin{aligned}
& \operatorname{Obj}\left(\theta_{D}, \theta_{G}\right)=-\int_{x} P_{\text {data }}(x) \log (D(x)) d x-\int_{Z} P_{Z}(Z) \log (1-D(G(Z))) d z \\
& =\int_{x}\left[P_{\text {data }} \log (D(x))+P_{G}(x) \log (1-D(x))\right] d x
\end{aligned}
$$

At this time, due to the data and generator have been given, they can be regarded as constants. Assuming that the data and generator are replaced, then

$$
f(D)=c_{1} \log D+c_{2} \log (1-D)
$$

Let $f(D)=0$ in (4), then it is the maximum point:

$D *(x)=\frac{P_{\text {data }}(x)}{P_{\text {data }}(x)+P_{G}(x)}$

The second step is to fix the discriminator $D$. At this time, the optimization function for the generator $G$ was calculated by using (6):

$$
V(G, D)=\mathrm{E}_{x \sim} P_{\text {data }} \log D(x)+\mathrm{E}_{x \sim} P_{G} \log [1-D(x)]
$$

Furthermore, using (5), $D *$ brings $V(G, D)$ as the optimal solution of the generator, which can be calculated by using (7):

$$
\begin{aligned}
& \min _{G} V(G, D)=V\left(G, D^{*}\right) \\
& =\mathrm{E}_{x \sim P_{\text {data }}}(x)\left[\log \frac{P_{\text {data }}(x)}{P_{\text {data }}(x)+P_{G}(x)}\right]+\mathrm{E}_{x \sim P_{G}}(x)\left[\log \frac{P_{\text {data }}(x)}{P_{\text {data }}(x)+P_{G}(x)}\right]
\end{aligned}
$$

In practical training, the discriminator $D$ is usually trained first. Then, the discriminator $D$ is fixed and the generator $G$ is trained. Next, we continue to fix $G$ and train the discriminator $D$, performing iterative optimization training until $P_{\text {data }}=P_{G}$, at which point global optimization is achieved.

\section{B. DESIGN OF THE STRUCTURE OF THE DCGAN}

Compared with traditional GANs, the salient feature of the DCGAN is that a CNN is used to replace the multilayer perceptron. The pooling layer and sampling layer are removed in the CNN model. The convolution layer is used to discriminate the image in the discriminator, and the deconvolution layer is used to generate the image in the generator. The specific structure of the DCGAN generator is as follows: the input layer is followed by a batch normalization layer (which can hasten the convergence of the model), and the reshaping layer is used to normalize the preliminary data; then, an upsampling layer, a 
Conv2DTranspose layer and a batch normalization layer are used to sample, deconvolute and normalize the data, respectively. In this paper, the DCGAN adds three groups of the above structures to increase the depth of the network. The main framework of the network architecture of the generator is shown in Figure 1.

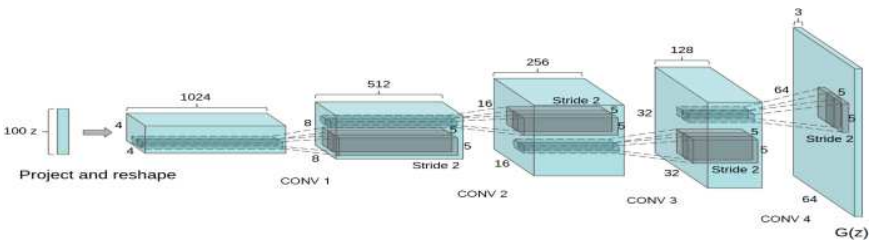

Figure 1. Network structure of the DCGAN generator [17]

In this paper, to solve the problem in which gradients disappear easily, the LeakyReLU activation function is used in all layers of the discriminator, and the Tanh activation function is used in the output layer of the generator, where definition of this function is (8):

$f(x)=\frac{1-e^{-2 x}}{1+e^{-2 x}}$

For the generator, except for the activation function of the last layer, the ReLU activation function is used, and its definition is (9):

$f(x)=\left\{\begin{array}{l}x, x \geq 0 \\ 0, x<0\end{array}\right.$

In addition, the concrete structure of the DCGAN discriminator is a Conv2D layer (2D convolution layer), a batch normalization layer and a dropout layer (after the image is convoluted, normalization process is continued, and the dropout layer is added to increase the generalization ability of the model). These three layers form a group, and four groups are added. Finally, a flattening layer and a fully connected layer are used to flatten the data and output the probability of whether it is sample data or generated data. Except for that of the last layer, the activation function of the other layers is the LeakyReLU function. The definition of this function is (10):

$f(x)=\left\{\begin{array}{l}x, x \geq 0 \\ a x, x<0\end{array}\right.$

where $x$ is a sample from the real data and $a$ is the relevant parameter.

The activation function of the last layer adopts the sigmoid function. The definition of this function is (11):

$\sigma(x)=\frac{1}{1+e^{-x}}$

III. CONSTRUCTION OF THE IMAGE GENERATION MODELS BASED ON THE DCGAN AND GANS

\section{A. DATA SOURCES}

This paper uses the MNIST dataset, which is free of charge, is open source, contains small pixels and includes a large number of points [18]. It is composed of 250 handwritten digits (0-9, a total of 10 digits), as shown in Figure 2

To universalize the dataset, $50 \%$ of the data in the dataset are from high school students, and $50 \%$ are from Census Bureau staff. At the same time, this paper uses the Keras framework with Tensorflow as the back end. The Keras framework is an open-source artificial neural network library written in Python. The code structure is written with an object-oriented method, which is completely modular and extensible. It is suitable for the implementation framework of the code in this experiment.
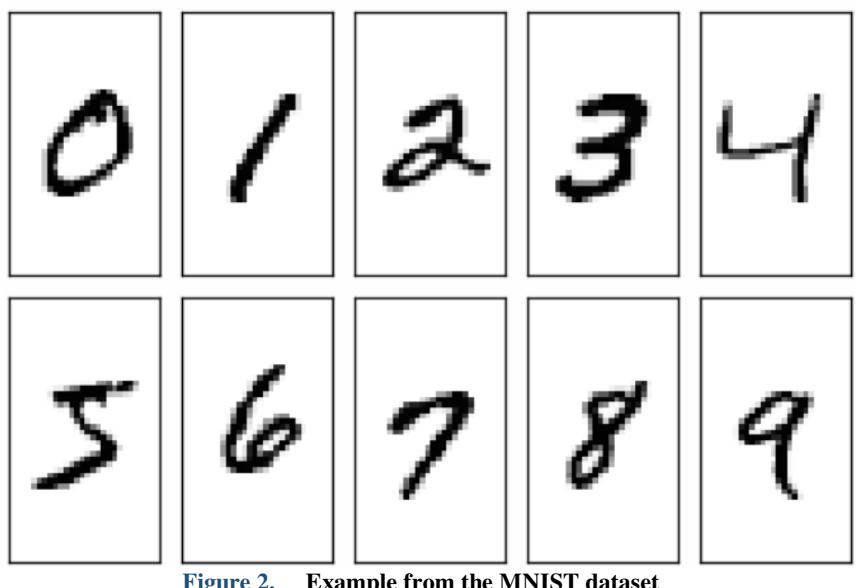

Figure 2. Example from the MNIST dataset

\section{B. DETERMINATION OF THE DCGAN MODEL PARAMETERS}

The model generator accepts $1 \times 100$ random, normallydistributed noise data. Considering that the layout of the pixels of the image generated by deconvolution is $28 \times 28$ (i.e., MNIST dataset image pixels), the input layer is set as a fully connected layer, and the number of neurons is $7 \times 7 \times 256$. After that, a batch normalization layer and reshaping layer are added, and then the size of the data pixel is expanded through the upsampling layer. Using a $5 \times 5$ convolution kernel, the conv2dspread layer uses the "same" border model to preserve the convolution results at the boundary, so that the input and output dimensions are the same. The upsampling layer is added after the first module to make the pixel size of the output image $28 \times 28$. The activation function in this layer uses the ReLU function, and the last layer uses the Tanh function.

In the discriminator, a Conv2D layer (2D convolution layer), a batch normalization layer and a dropout layer are added as a module, and four modules are added. Finally, a flattening layer and a fully connected layer are used as the back end. The Conv2D layer uses a $5 \times 5$ convolution kernel, the boundary mode of the convolution layer is the same as that in the generator, and the activation function is the LeakyReLU function. The dropout layer in the module makes the activation value of a certain neuron have a certain 
probability $\mathrm{p}$ when it propagates forward. This can make the model independent of some local features and enhance the generalization ability of the model. The last activation function uses the sigmoid function, which can output the probability of discrimination, that is, the probability that the discriminator thinks the image belongs is the real image and not a generated image.

The discriminator uses the Adam optimizer with a learning rate of 0.0002 , and the GANs use the RMSprop optimizer with a learning rate of 0.0001 . The RMSprop optimizer combines the exponential moving average of the square of the gradient to adjust the learning rate. It can converge effectively under an unstable objective function and yields good results with the DCGAN model. The batch size is 32 , and the training time of each DCGAN iteration is 10000. The binary cross-entropy function is selected as the loss function.

The training process of the DCGAN is slightly different from that of the GANs because it takes more time to train the discriminator of the DCGAN model. First, when training the discriminator, the input data size is $2 \times$ batch, where the input data contains the real data and generated data from one batch; second, the combined data of size $2 \times$ batch are used as the input data to train the discriminator 5 times; finally, the whole GAN model is trained once with the random noise data from one batch as the input data, and only the generator is updated. A complete training cycle is a batch (epoch) in which the ratio of discriminator training iterations to generator training iterations is 1:5 to realize the alternating iterative training process. The main parameter configuration of the DCGAN is shown in TABLE 1.

TABLE 1

MAIN PARAMETERS OF THE DCGAN

\begin{tabular}{cc}
\hline \hline Parameter & Value \\
\hline BATCHSIZE & 32 \\
EPOCH & 100,000
\end{tabular}

LEARNINGRATE(GANs)

DECAY(GANs)

LEARNINGRATE(Discriminator)

DECAY(Discriminator)

ALPHA(LeakyReLU)

MOMENTUM(BatchNormalization)

DROPOUT

0.0001

0.00000003

0.0002

0.00000006

0.2

0.9

0.3

STRIDES(Conv2D)

2

OUTPUTWIDTH

28

OUTPUTHIGHT

28

OUTPUTCHANNEL

\section{DETERMINATION OF THE PARAMETERS OF THE GAN MODEL}

The noise data received by the generator are the same as above, and a basic multilayer perceptron is used to add these three modules as a fully connected layer, a LeakyReLU layer and a batch normalization layer; the model ends with a fully connected layer and a reshaping layer. The activation function of the last layer uses the Tanh function.

The discriminator uses a flattening layer to flatten the data and then adds two fully connected layers. The activation function also uses the LeakyReLU function, and the last activation function uses the sigmoid function to output the discrimination probability. Both the GANs and the discriminator use Adam as their optimizer, and the learning rate is 0.002 . To calculate the update step size, the Adam optimizer comprehensively considers the first-order moment estimation (average value of the gradient) and second-order moment estimation (noncentral variance of the gradient). The batch size is 32. Due to the slow convergence speeds of GANs, the model can avoid falling into local optimal solutions and undergo training $100 \mathrm{~K}$ times iteratively. The binary cross-entropy function is selected as the loss function. The main parameter configuration of the GANs is shown in TABLE 2.

TABLE 2

MAIN PARAMETERS OF THE GANS

\begin{tabular}{cc}
\hline \hline Parameter & Value \\
\hline BATCHSIZE & 32 \\
EPOCH & 100,000 \\
LEARNINGRATE(GANs) & 0.0002 \\
LEARNINGRATE(Discriminator) & 0.0002 \\
DECAY(Discriminator) & 0.000000009 \\
ALPHA(LeakyReLU) & 0.2 \\
MOMENTUM(BatchNormalization) & 0.8 \\
OUTPUTWIDTH & 28 \\
OUTPUTHIGHT & 28 \\
OUTPUTCHANNEL & 1 \\
LATENTSIZE & 100 \\
\hline \hline
\end{tabular}

During training, the discriminator is trained once, and the input data are half true and half false, i.e., the input dataset is composed of half real data and half batch-generated data. Such a complete combined dataset is used as input data to train the discriminator once. Then, the whole GAN model is trained once with a batch of random noise data as input, and only the generator is updated. Such a training cycle is a batch (epoch), which is performed to realize the alternating iterative training process.

\section{EXPERIMENTAL RESULTS OF THE IMAGE GENERATION MODELS BASEDE ON THE GANS AND DCGAN}

There are experimental groups in the training experiments of the image generation models based on the GANs and DCGAN. Each experimental group is divided into Experiment 1 (composed of 250 handwritten digits (0-9, a total of 10 digits)) and Experiment 2 (only using the number 
"6" in the dataset). The experiment under the unified experimental group is conducted to observe the learning effects of different networks with different image distribution complexity and image generation quality; the experiment with different experimental groups using the same dataset is done to compare the advantages and disadvantages of the GANs and DCGAN with regard to image generation.

1) Training results of the image generation model based on the DCGAN:

Experiment 1: Set checkpoints in the training process, run the training process for $10 \mathrm{~h}$ in the local environment, and observe the output results once every 50 training iterations. The results are shown in Figure 3, in which Figure 3 (a), 3(b), $3(\mathrm{c})$ and $3(\mathrm{~d})$ are the results of the original noise image, the results after $1 \mathrm{~K}$ training iterations, the results after $5 \mathrm{~K}$ training iterations and the results after $10 \mathrm{~K}$ training iterations, respectively. By observing the output at each checkpoint, we can find that the original noise data are disordered. After $1 \mathrm{~K}$ iterations, the image is gradually regionalized, and the only content is in the central area. However, most of the digital contours cannot be clearly recognized, and obvious characteristics of the deconvolution layer can be found. The image learning process includes regionalization and characterization rather than pixel learning, similar to the process of the GANs. Training $5 \mathrm{~K}$ times can yield a gradually clearer line for which the numbers can be identified but not recognized clearly. After training $10 \mathrm{~K}$ times, each number can be clearly identified, clear images are generated, and the complex image distribution is successfully fitted.
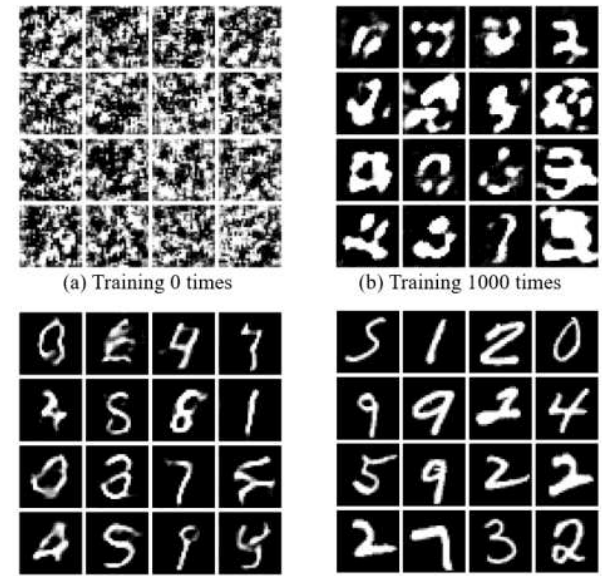

(c) Training 5000 times

(d) Training 10000 times

Figure 3. DCGAN partial results (Experiment 1)

From the loss images of the discriminator (Figure 4(a)) and the generator (Figure 4(b)), it can be seen that the discriminator stabilizes at approximately 0.5 for a very short batch. For the $1 \mathrm{~K}$ batch, the loss of the generator decreases to 2 , then approaches 1 slowly, and finally stabilizes at approximately 1.5 , but there is a downward trend. Because of this configuration, the experiment cannot continue; even if the Nash equilibrium point is not reached, the effect of the output image is still very good, and this shows that the DCGAN can obtain better experimental results than those of other methods under the premise of satisfying the computational power requirements.

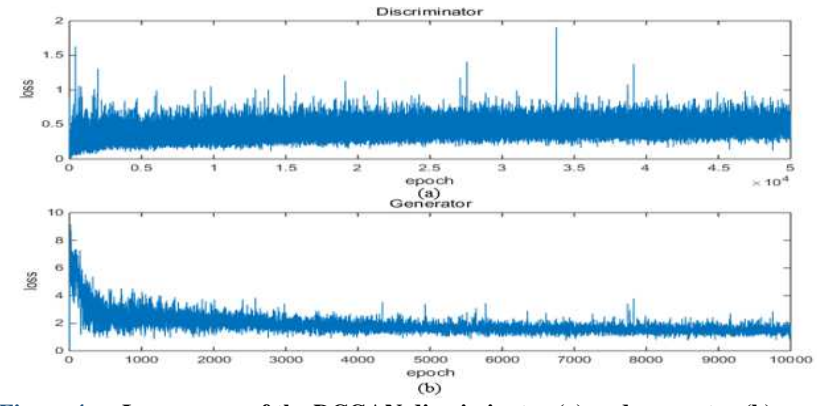

Figure 4. Loss curves of the DCGAN discriminator (a) and generator (b) (Experiment 1)

Experiment 2: run the same training process as in Experiment 1 in the local environment for $10 \mathrm{~h}$; the results are shown in Figure 5, in which Figure 5(a), 5(b), 5(c) and 5(d) are the results of original noise image, the results after training $1 \mathrm{~K}$ times, the results after training $5 \mathrm{~K}$ times and the results after training $10 \mathrm{~K}$ times, respectively. By observing the output of each checkpoint, it can be found that the original noise is the same as in Experiment 1. When iterating $1 \mathrm{~K}$ times, the number " 6 " can be identified, such as the first number from the left in the second row and the fourth number from the left in the third row; most of the number "6" can be recognized after iterating $5 \mathrm{~K}$ times. All the numbers can be recognized after $10 \mathrm{~K}$ iterations, but the numbers are not standardized, mainly because the MNIST dataset contains handwritten numbers from adults and children.

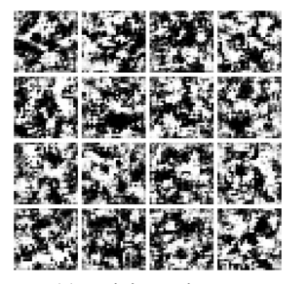

(a) Training 0 times

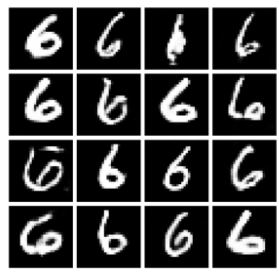

(c) Training 5000 times

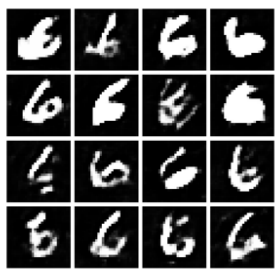

(b) Training 1000 times

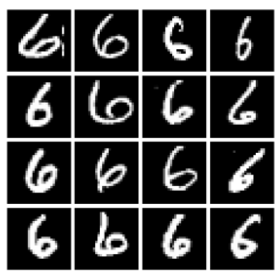

(d) Training 10000 times
Figure 5. Partial results of the DCGAN (Experiment 2)

According to the loss images of the discriminator (Figure 6(a)) and generator (Figure 6(b)), the discriminator D starts to fluctuate at approximately 0.5 at 1000 epochs (the ratio of the training times of the discriminator and generator is $5: 1$ ); the generator also starts to stabilize at approximately 2 at $1 \mathrm{~K}$ epochs, gradually converges to 1 , and finally fluctuates at approximately 1 with a small fluctuation range. 


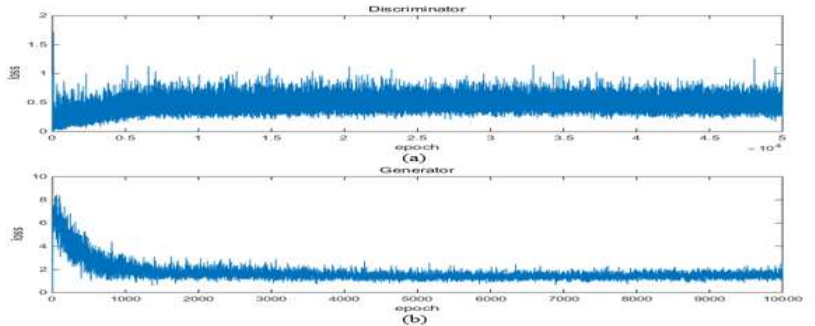

Figure 6. Loss curves of the DCGAN discriminator (a) and generator (b) (Experiment 2)

Through the comparative training processes of the two experiments, it is found that the DCGAN model can adapt to both complex image distributions and simple image distributions, and it can converge earlier than other methods. Its learning law is characterized and regionalized. At the beginning, it concentrates on the central area, then learns the line features, and finally learns the location characteristics of the lines.

2) Training results of the image generation model based on GANs

Experiment 1: Set checkpoints in the training process, run the training process for $5 \mathrm{~h}$ in the experimental environment, and observe the output results once every 500 training iterations. The results are shown in Figure 7, in which Figure 7(a), 7(b), 7(c), and 7(d) are the results of the original noise image, the results after training $10 \mathrm{~K}$ times, the results after training $50 \mathrm{~K}$ times and the results after training $100 \mathrm{~K}$ times, respectively. By observing the output at each checkpoint, we can find that the original noise data are disordered. After $10 \mathrm{~K}$ iterations, the image is gradually focused in the central area rather than at scattered points in each position. After iterating $50 \mathrm{~K}$ times, some figures have a preliminary outline, indicating that the generator is gradually learning the image distribution of the original dataset. Continuing to train for a total of $100 \mathrm{~K}$ iterations, one can find that most of the figures are clear and distinguishable. This situation lasts nearly $20 \mathrm{~K}$ batches during the training process, indicating that the GANs maintain stability for a long time but only reach the pseudoNash equilibrium point. It is found that adding a hidden layer does not affect the experimental results but rather increases the experimental time.

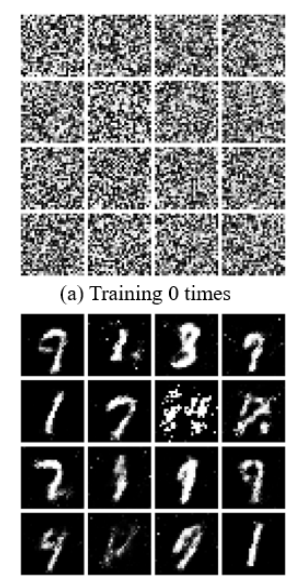

(c) Training 50000 times
Figure 7. Partial results of the GANs (Experiment 1)

By observing the loss function images of the discriminator (Figure 8(a)) and generator (Figure 8(b)), it is not difficult to see that the loss of the generator decreases rapidly in each of the first $10 \mathrm{~K}$ batches, then gradually approaches 1 , fluctuates greatly between $30 \mathrm{~K}$ to $70 \mathrm{~K}$ iterations, and finally stabilizes near 1 . This shows that the image generated by the generator can make the discriminator think that it is true. However, the discriminator gradually becomes stable at approximately 0.5 starting with the 15000th batch, and then it fluctuates up and down. As the number of batches increases, the fluctuation range does not decrease, and the overall loss is slightly less than 0.5, indicating that the discriminator has a high probability of correct discrimination (recognition of the real image). After $100 \mathrm{~K}$ iterations, the Nash equilibrium point cannot be reached, but the model cannot be further converged.

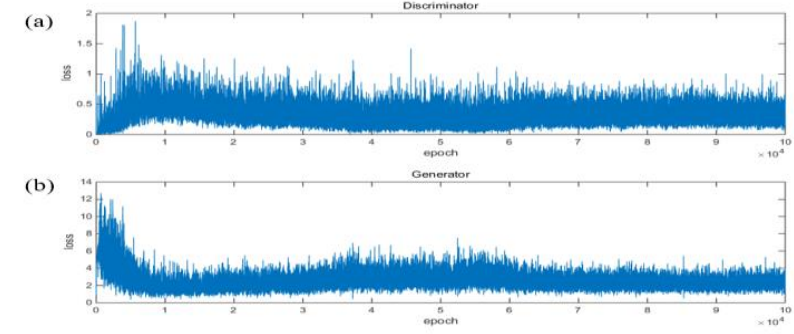

Figure 8. Loss curves of the GANs discriminator (a) and generator (b) (Experiment 1)

Experiment 2: The running time of the training process is $5 \mathrm{~h}$ in the local environment, and the results are shown in Figure 9, where Figure 9(a), 9(b), 9(c) and 9(d) are the results of the original noise image, the results after training $10 \mathrm{~K}$ times, the results after training $50 \mathrm{~K}$ times and the results after training $100 \mathrm{~K}$ times, respectively. The original noise is the same as in Experiment 1, and the learning process of the GANs can still be seen in (b) and (c), but the speed of the pixel setting is much higher than that in Experiment 1. Compared with Figure 9(d) and Figure 7(d) of Experiment 1, it can be found that when iterating $100 \mathrm{~K}$ times, GANs can effectively fit the simple image distribution. The second digit on the left in the second row of Figure 7(d) generates "6", but this is not as effective as simply learning "6". In Figure 9(d) of Experiment 2, all the numbers "6" can be clearly identified. 


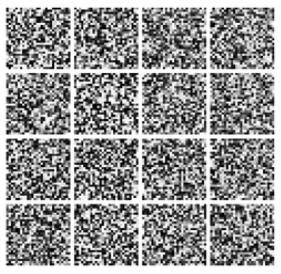

(a) Training 0 times

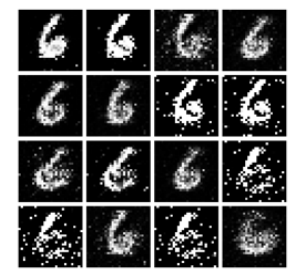

(b) Training 10000 times

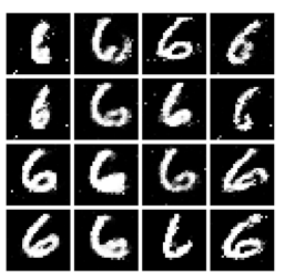

(c) Training 50000 times

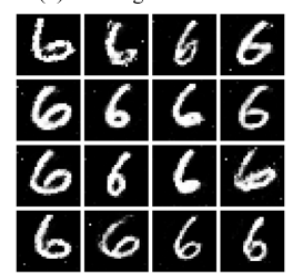

(d) Training 100000 times
Figure 9. Partial results of the GANs (Experiment 2)

Comparing the loss function images of the discriminator (Figure 10(a)) and generator (Figure 10(b)), it is not difficult to find that the generator loss rapidly decreases in each of the first $20 \mathrm{~K}$ batches, gradually approaches 1 , and stabilizes near 1. This shows that the image gradually generated by the generator can make the discriminator think it is true. Since the 20000th batch, the discriminator gradually stabilizes at approximately 0.5 and gradually reduces its fluctuation range, but the overall loss is slightly higher than 0.5 , which indicates that the discriminator cannot correctly judge that the generated image is real, and the loss curve still cannot converge at 0.5 ; that is, it cannot reach the real Nash equilibrium point.

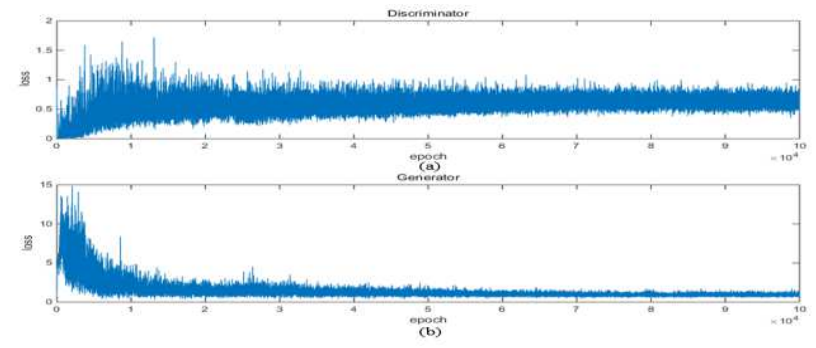

Figure 10. Loss curves of the GANs discriminator (a) and generator (b) (Experiment 2)

\section{COMPARATIVE ANALYSIS AND ASSESSMENT OF IMAGE GENERATION QUALITY}

\section{A. CONSTRUCTION OF THE EVALUATION INDEX FOR IMAGE GENERATION}

When comparing and analyzing the quality of images generated by models, two factors are generally considered: one is the textures of the images, and the other is the diversity of image generation. At present, the popular quantitative evaluation method for image texture is the inception score (IS). In this paper, the simple initial fraction (ISs) is used to evaluate image quality, and the complex initial fraction (ISc) is used to evaluate image diversity.

\section{B. IMAGE QUALITY ASSESSMENT}

The calculation formula of the ISs evaluation index using the simple initial score method is (12):

$$
I S_{E}(G)=\exp \left(\mathrm{E}_{x \sim P_{G}} D_{K L}(p(y \mid x) \| p(y))\right)
$$

where $x \sim P_{G}$ represents the image generated by the generator to be evaluated, $p(y \mid x)$ represents the probability that the picture belongs to each category, and $p(y)$ is the probability distribution of the image to be evaluated.

The following formula can be obtained by further derivation (13):

$$
\operatorname{In}\left(I S_{E}(G)\right)=H(y)-H(y \mid x)
$$

It can be seen from the (13) that the larger the ISs evaluation index is, the greater the differences between the $p(y \mid x)$ and $p(y)$ distributions.

If only the ISs comprehensive analysis method is used, there may be a large error. In this paper, a concept classification network is proposed to eliminate the error as much as possible. In the MNIST dataset, there are 10 numbers from 0 to 9 , and each image is a black and white image (the value is composed of $0 \mathrm{~s}$ and 1s). For any two random variables $X, Y$, the correlation coefficient is calculated as follows:

$$
\tau(X, Y)=\frac{\operatorname{Cov}(X, Y)}{\sqrt{\operatorname{Var}[X] \operatorname{Var}[Y]}}
$$

where $\operatorname{Cov}(X, Y)$ in (14) is the covariance of $X$ and $Y$, $\operatorname{Var}[X]$ is the variance of , and $\operatorname{Var}[Y]$ is the variance of $Y$.

According to the principle of the concept classification network, it is not difficult to know that if the image correlation is weak, the classifier easily divides it into two categories; otherwise, it is easier to divide it into one category. When considering the diversity of images, we should consider the distribution of the labels. If the distribution of complex images is complex, we naturally want the labels to be evenly distributed instead of generating a certain kind of image. For example, when all the numbers in the MNIST dataset are used, a good situation is that the generated $0 \mathrm{~s}-9 \mathrm{~s}$ are evenly distributed, rather than the distribution containing more of certain numbers than others. In this case, we need to consider the edge probability $p(y)$. In the ideal state, the expansion can yield $p\left(y_{1}\right)=p\left(y_{2}\right)=\cdots=p\left(y_{n}\right)=1 / n$, where $n$ is the number of classes in the original training data; the greater the entropy of $p(y)$, the better the situation is.

If the same kind of data are used, that is, a single number is used, although these data would be highly correlated and belong to the same class, because of the characteristics of the concept classification network, the set would still be divided into several categories, but $\lim p\left(y_{1}\right)=1$ and $\lim p\left(y_{i}\right)=0$ where $i=2,3, \cdots, n$. At this time, $n$ is 
the number of classification categories for the concept classification network.

\section{IMAGE DIVERSITY ASSESSMENT}

The complex initial fraction (ISc) is based on the simple initial fraction method. According to the image classification value of the simple distribution, the entropy of the simple image distribution under discrete conditions is calculated, where $H$ in (15) represents entropy, and the calculation formula is as follows:

$$
H\left(p\left(y_{i}\right)\right)=\sum_{i=1}^{n} p\left(y_{i}\right) \times \log \left(p\left(y_{i}\right)\right)
$$

The edge probability value of the simple image distribution is included when $i=1$, using (16), we calculated the potential:

$$
H\left(p\left(y_{i}\right)\right)=H\left(p\left(y_{1}\right)\right)=0
$$

When $i=2,3, \cdots, n, \lim p\left(y_{i}\right)=0^{+}$:

$H\left(p\left(y_{i}\right)\right)=\sum_{i=1}^{n} p\left(y_{i}\right) \times \log \left(p\left(y_{i}\right)\right)=0$

Thus, the entropy limit of the concept classification network is obtained when the image tends to be simple distribution, i.e., when $\lim H(p(y))=0$ in (17). Then, the formula of the ISc evaluation index becomes:

$$
\operatorname{In}\left(I S_{C}(G)\right)=H(y \mid x)
$$

where $H(y \mid x)$ in (18) represents the probability that an image belongs to a certain category. The higher the value is, the higher the image quality. Therefore, when the image distribution is simple, the ISc under a complex distribution can be used to comprehensively evaluate the diversity of the images. The diversity of image generation can be combined with two experiments and a comprehensive ISc analysis. The two groups of experiments discuss image generation under different complexities of the image distribution. If the ISc value can still reach a high value under the condition of a complex image distribution, this shows that the diversity of image generation is very high.

\section{COMPARATIVE ANALYSIS OF AXPERIMENTAL RESULTS}

According to the image quality evaluation method proposed in Section 4.1, two groups of experiments are compared and analyzed. For the MNIST dataset, first, 10K groups of images are generated by the generator, all data are divided into 10 pieces, and each piece is calculated and averaged. Second, the concept classification network is built to calculate the ISc and ISs of the experimental GANs and the DCGAN, respectively. The experimental results are shown in TABLE 3 . The first experiment is conducted to evaluate the image diversity, and the second is performed to evaluate the image quality.

TABLE 3

EXPERIMENTAL RESULTS OF THE IMAGE QUALITY COMPARISON

\begin{tabular}{ccc}
\hline \hline Experimental Group & Model & IS $(28 \times 28)$ \\
\hline \multirow{2}{*}{ Group 1 } & GANs & ISC: 4.55 \\
& DCGAN & ISC: 6.10 \\
\multirow{2}{*}{ Group 2 } & GANs & ISE: 4.80 \\
& DCGAN & ISE: 6.82 \\
\hline \hline
\end{tabular}

According to the experimental results in TABLE 3, in terms of image quality, the ISs value of the DCGAN model is 2.02 higher than that based on the GANs model; in terms of image generation diversity, the ISc value based on the DCGAN model reaches 6.10 , which is 1.55 higher than that based on the GANs model. The results show that the improved DCGAN model has more advantages than the GANs model, can effectively solve the problem of low-quality images being output by the GANs model, and achieves good results.

\section{DISCUSSION AND CONCLUSIONS}

Based on the GANs model and the improved DCGAN model, this paper uses the MNIST dataset as experimental data for experiments on the algorithms and evaluates the quality and diversity of the generated images based on the ISs and ISc metrics.

1) This paper compares and analyzes the different performances of traditional GANs and the DCGAN in two groups of experiments.

The DCGAN is a model based on a combination of GANs and a convolutional neural network. The fully connected layer is replaced by a convolution layer and deconvolution layer. The structure of the DCGAN layers is redesigned: An upsampling layer is used in the output layer of the generator to expand the data, and a dropout layer is added in each layer of the discriminator. To solve the problem that the gradient easily disappears in GANs, the generator output process uses a beneficial Tanh function; the ReLU function is used in the other layers of the generator, and the LeakyReLU function is used in all layers of the discriminator. In the two groups of comparative experiments, it can be concluded that the images of the GANs model are generated in a column because of the flattening layers and reshaping layers. The image generation method using the DCGAN constructed in this paper involves generating regional and characteristic images by using a conv2dspread layer (twodimensional anti-convolution layer), which fundamentally solves the problem of low-quality images being generated by the GANs.

2) In this paper, through the optimized DCGAN model, it is proven that the variables of a simple image distribution tend to be independent of each other, thereby overcoming the error caused by the independence of traditional indexes.

The ISs value under the simple image distribution is taken as the image quality evaluation result, and the ISc 
value of the complex image distribution is combined with it to comprehensively evaluate the diversity and quality of the generated image. Through two groups of experiments, it can be concluded that the image quality evaluation index ISs of the DCGAN model is 6.82, which is 2.02 higher than that of the GANs model with the same image distribution. The image diversity index ISc of the DCGAN is 6.10 , while that of the GANs is only 4.55 . The reason for this is that the image quality evaluation index ISs of the DCGAN is 6.82, which is 2.02 higher than that of the GANs model with the same image distribution. In addition, the DCGAN has more advantages than the GANs in terms of its model framework and model detail parameters.

\section{DECLARATIONS:}

Ethical approval

There are no ethical issues in this paper.

Funding details (In case of Funding)

This work was supported in part by the National Key R\&D Program of China under Grant 2018YFC0604105, in part by National Natural Science Foundation of China under Grant 42072322, and Chengdu University of Technology Development Funding Program for Young and Middle-aged Key Teachers under Grant 10912-JXGG2020-06251.

\section{Conflicts of Interest}

All authors declare that there is no conflict of interest regarding the publication of this manuscript.

\section{Informed Consent}

The authors of this paper are informed and participate in the writing of the paper.

\section{Authorship contributions}

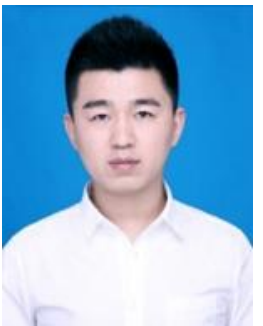

Bingqi Liu, male, 31 years old, Ph.D., graduated from Chengdu University of Technology, currently working at Chengdu University. Currently, he is mainly engaged in research based on image processing and computer data extraction.

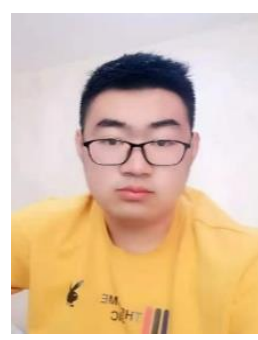

Jiwei Lv, male, 22 years old. graduated from Chengdu University of Technology. Mainly engaged in research based on graphics processing model improvement and computer big data processing.

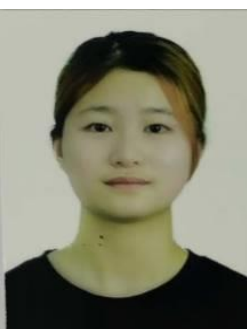

Xinyue Fan, female, 22 years old, is a doctoral student at Chengdu University of Technology. Mainly engaged in research on management science and engineering. At present, mainly engaged in the research of deep-level information mining based on image processing and machine learning.

Jie Luo, male, 22 years old, majored in applied

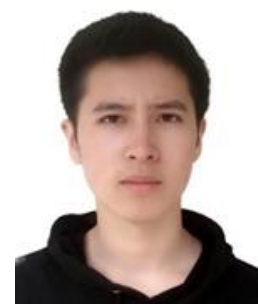
statistics from Guangxi University of science and technology, School of management science, Chengdu University of technology. At present, mainly studying deep-seated ore prospecting research based on big data and deep learning.

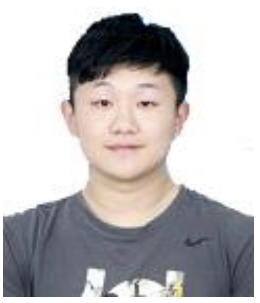

Tianyi Zou, male, 25 years old, majored in applied statistics in the college of management science, Chengdu University of technology, and graduated from Southwest University of Finance and Economics. At present, the research direction is big data technology and application, focusing on machine learning and mineral prediction.

\section{REFERENCES}

[1] A. V. D. Oord, N. Kalchbrenner, and K. Kavukcuoglu, "Pixel recurrent neural networks," In ICML'16 Proceedings of the 33rd International Conference on International Conference on Machine Learning., vol. 48, pp. 1747-1756, 2016.

[2] D. P. Kingma, and, M. Welling, "Auto-Encoding Variational Bayes," In ICLR 2014: International Conference on Learning Representations (ICLR)., 2014.

[3] I. J. Goodfellow, J. P. Abadie, M. Mirza, B. Xu, D. W. Farley, and, S. Ozair, "Generative adversarial networks," Advances in Neural Information Processing Systems., vol. 3, pp. 2672-2680, 2014.

[4] M. Mirza, and, S. Osindero, "Conditional Generative Adversarial Nets," ArXiv Preprint ArXiv., no. 1411, pp. 1784, 2014.

[5] T. Salimans, I. Goodfellow, W. Zaremba, V. Cheung, A. Radford, and X. Chen, "Improved techniques for training GANs," In NIPS'16 Proceedings of the 30th International Conference on Neural Information Processing Systems., pp. 2234-2242, 2016.

[6] P. Isola, J. Y. Zhu, T. Zhou, and A. A. Efros, "Image-to-Image Translation with Conditional Adversarial Networks," In 2017 IEEE Conference on Computer Vision and Pattern Recognition (CVPR)., pp. 5967-5976, 2017.

[7] H. Zhang, T. Xu, and, H. Li, "StackGAN: Text to Photo-Realistic Image Synthesis with Stacked Generative Adversarial Networks," In 2017 IEEE International Conference on Computer Vision (ICCV)., pp. 5908-5916, 2017.

[8] J. Y. Zhu, T. Park, P. Isola, and A. A. Efros, "Unpaired Image-to-Image Translation Using Cycle-Consistent Adversarial Networks," In 2017 IEEE International Conference on Computer Vision (ICCV), pp. 
2242-2251, 2017.

[9] T. Karras, T. Aila, S. Laine, and J. Lehtinen, "Progressive Growing of GANs for Improved Quality, Stability, and Variation," In ICLR 2018 : International Conference on Learning Representations., 2018.

[10] X. Huang, and S. Belongie, "Arbitrary Style Transfer in Real-Time with Adaptive Instance Normalization". In 2017 IEEE International Conference on Computer Vision (ICCV)., pp. 1510-1519, 2017.

[11] A. Brock, J. Donahue, and K. Simonyan, "Large Scale GAN Training for High Fidelity Natural Image Synthesis," In ICLR 2019: 7th International Conference on Learning Representations., 2019.

[12] A. Radford, L. Metz, and S. Chintala, "Unsupervised Representation Learning with Deep Convolutional Generative Adversarial Networks," In ICLR 2016: International Conference on Learning Representations., 2016.

[13] S. Liu, M. Yu, M. Li, and Q. Xu, "The research of virtual face based on Deep Convolutional Generative Adversarial Networks using TensorFlow," Physica A-Statistical Mechanics and Its Applications., vol. 521, pp. 667-680, 2019.

[14] M. A. B. Mahmoud, and P. Guo, "A Novel Method for Traffic Sign Recognition Based on DCGAN and MLP With PILAE Algorithm," IEEE Access., vol. 7, pp. 74602-74611, 2019.

[15] W. Fang, Y. Ding, F. Zhang, and J. Sheng, "Gesture Recognition Based on CNN and DCGAN for Calculation and Text Output," IEEE Access., vol. 7, pp. 28230-28237, 2019.

[16] S. Aslan, U. Gudukbay, B. U. Toreyin, and A. E. Cetin,. "Early Wildfire Smoke Detection Based on Motion-based Geometric Image Transformation and Deep Convolutional Generative Adversarial Networks," In ICASSP 2019 - 2019 IEEE International Conference on Acoustics, Speech and Signal Processing (ICASSP.)., pp. 83158319, 2019.

[17] J. Viola, Y. Q. Chen, and J. Wang, "Faultface: deep convolutional generative adversarial network (DCGAN) based ball-bearing failure detection method," Information Sciences, vol. 542, no. 4, pp. 195-211, 2020.

[18] K. Y. Cheng, R. Tahir, L. K. Eric, and M, Z. Li, "An analysis of generative adversarial networks and variants for image synthesis on MNIST dataset," Multimedia Tools and Applications, vol. 79, pp. 3725-13752, 2020. 


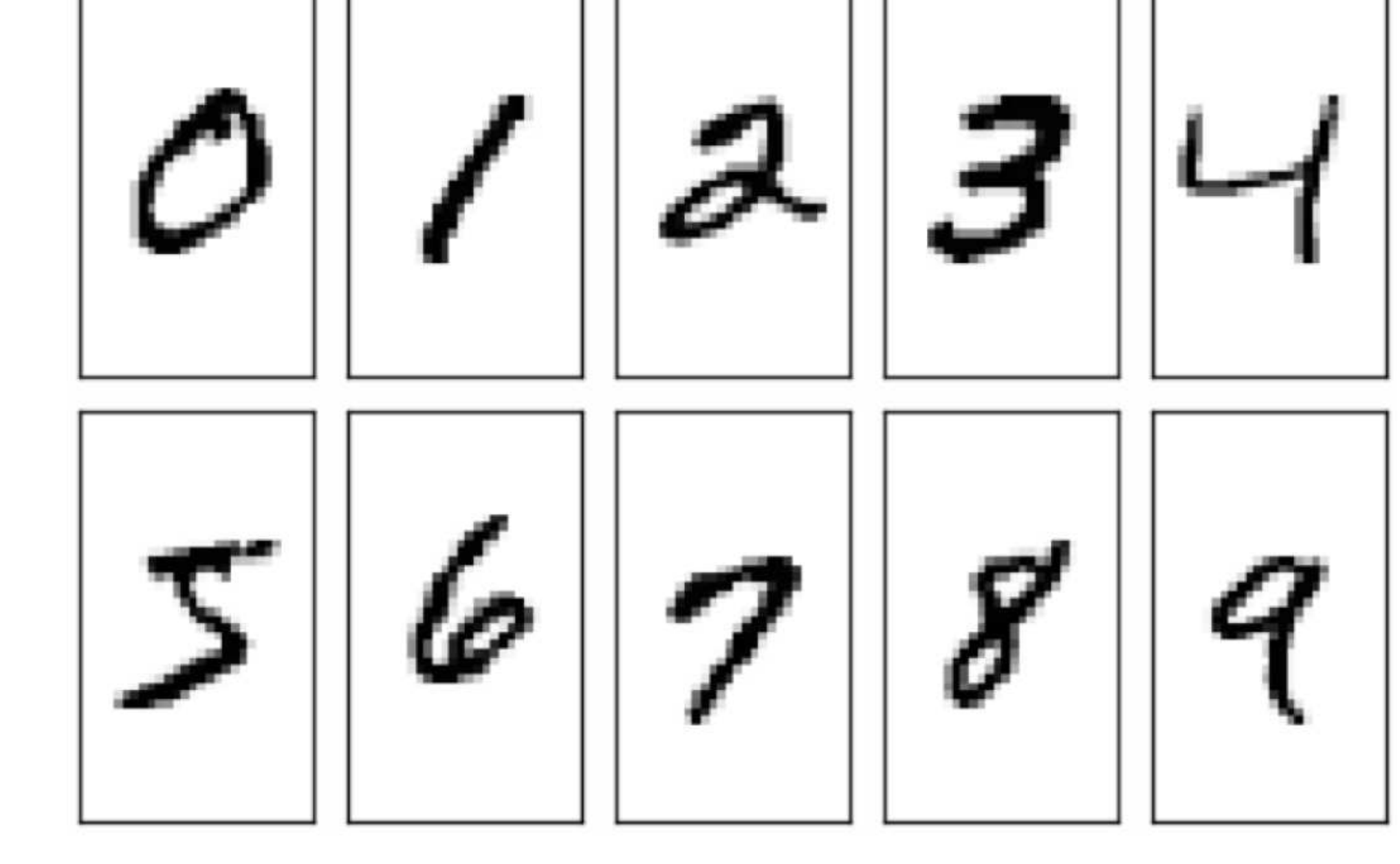




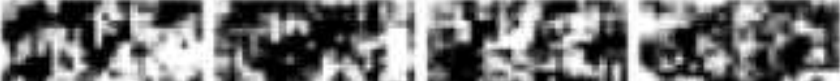

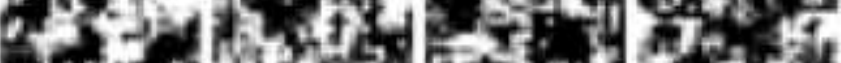

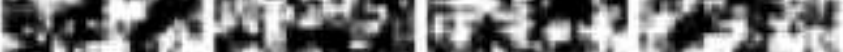

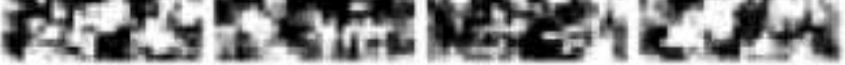
r

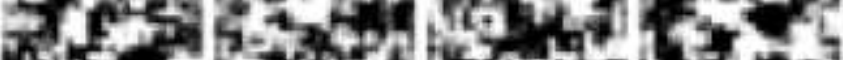

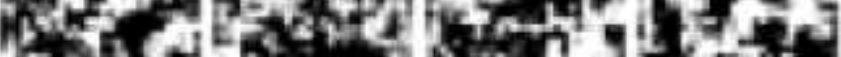

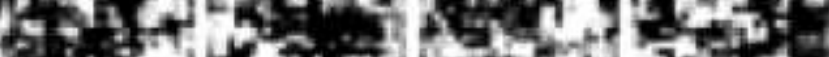

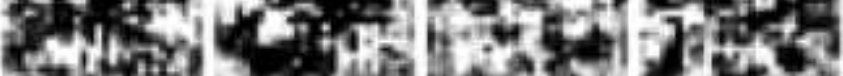

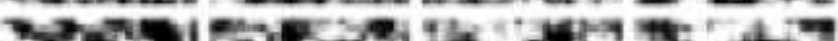

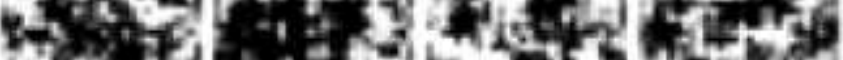

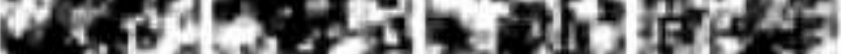

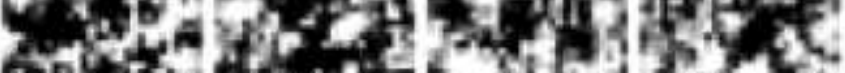

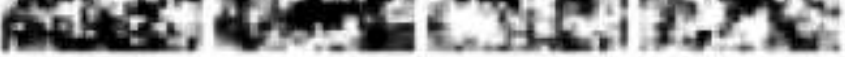
If

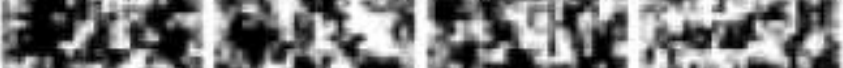

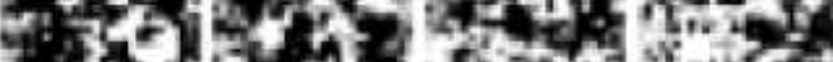

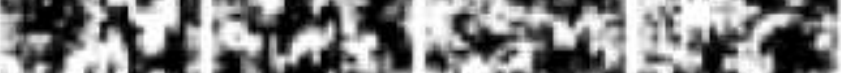

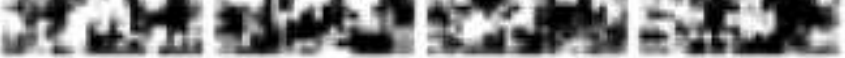

(a) Training 0 times
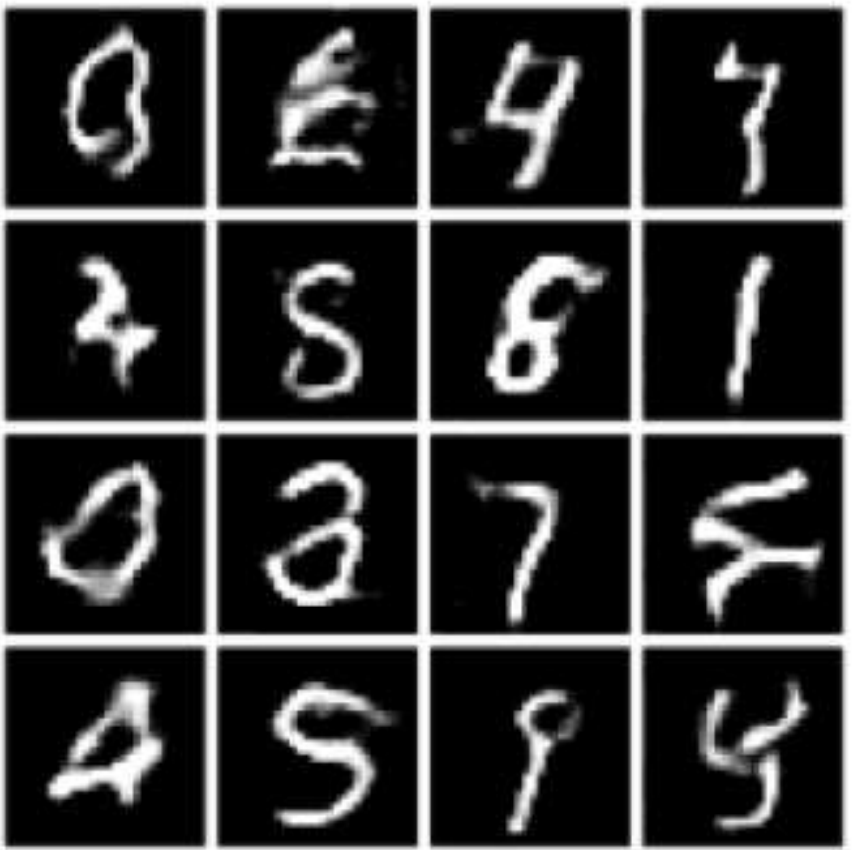

(c) Training 5000 times
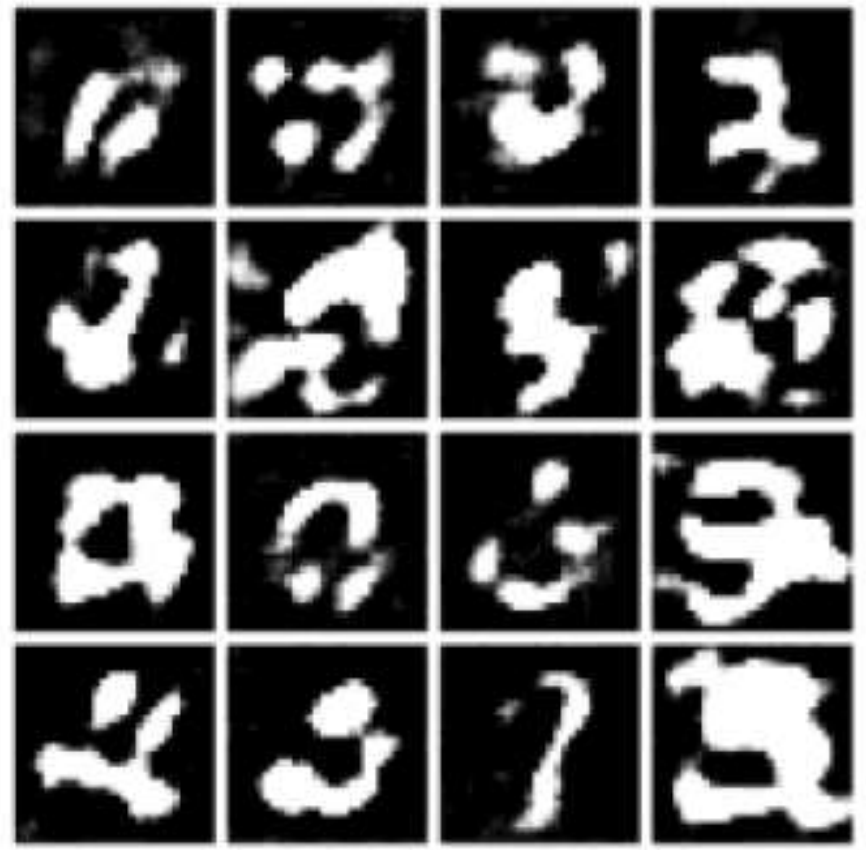

(b) Training 1000 times
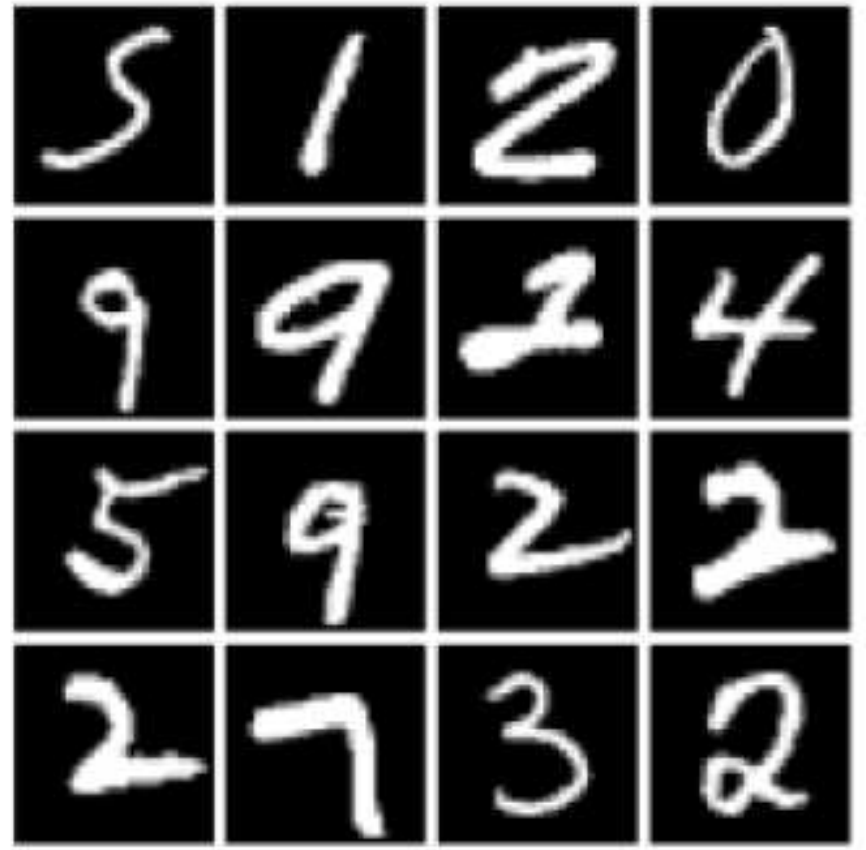

(d) Training 10000 times

Figure 3

DCGAN partial results (Experiment 1) 


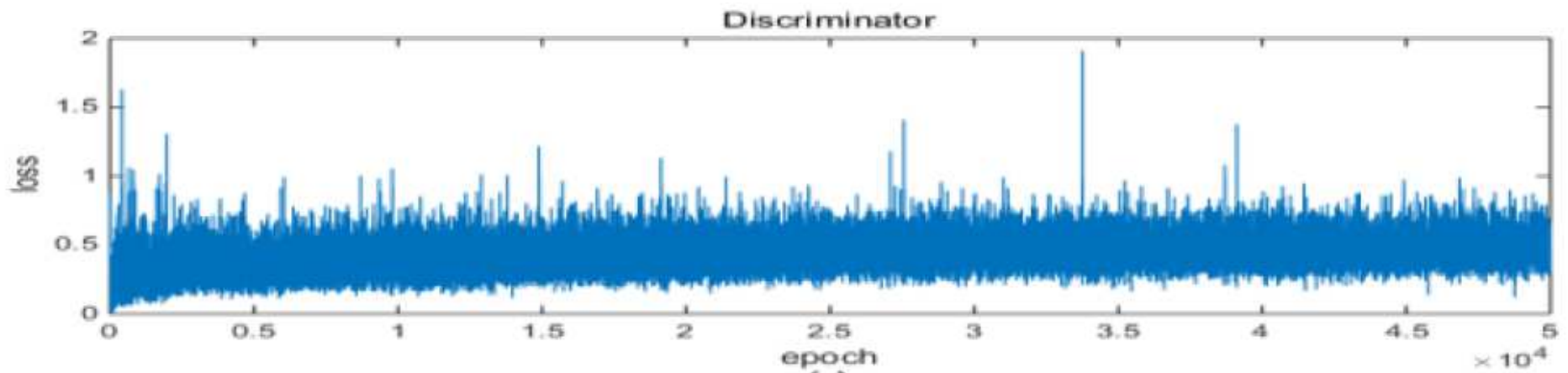

(a)

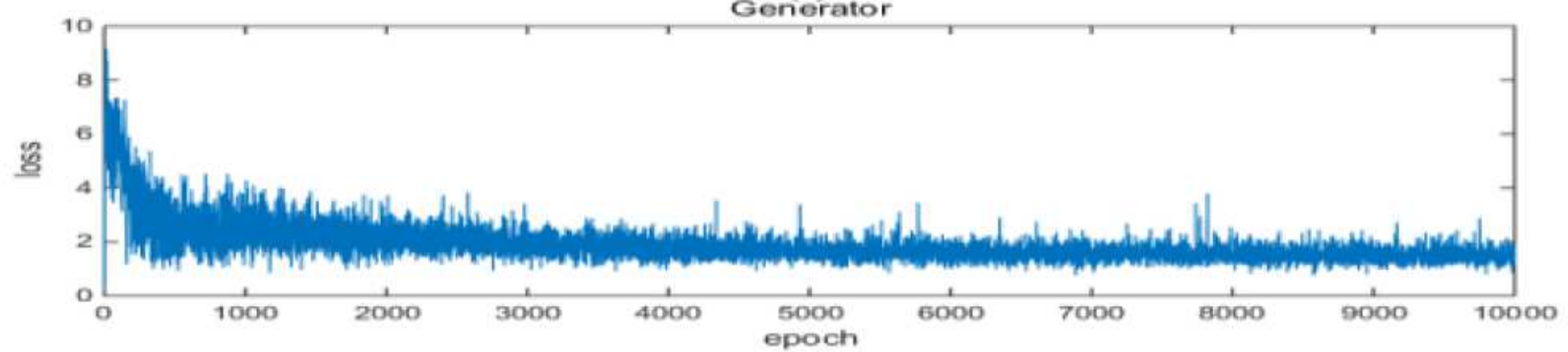

(b)

\section{Figure 4}

Loss curves of the DCGAN discriminator (a) and generator (b) (Experiment 1) 


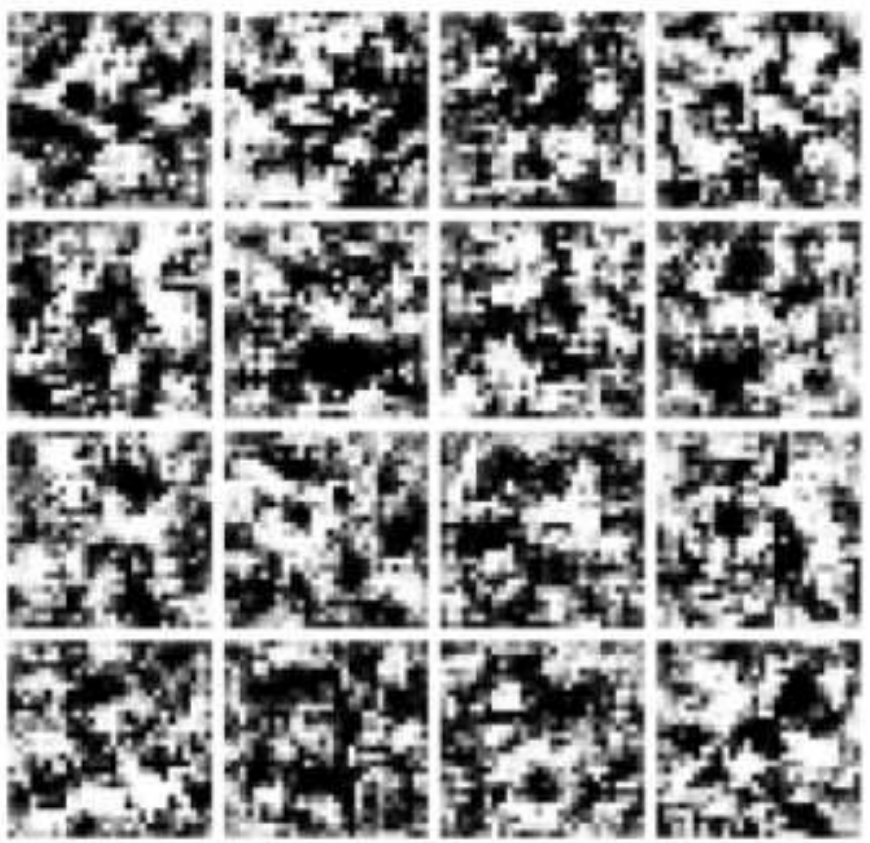

(a) Training 0 times

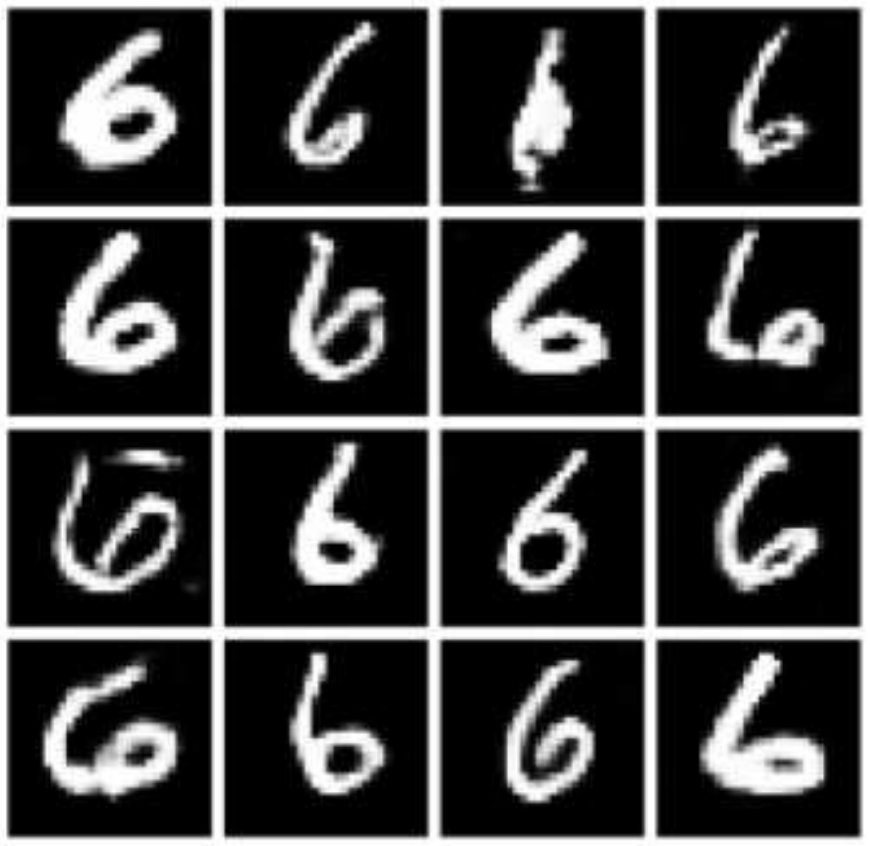

(c) Training 5000 times
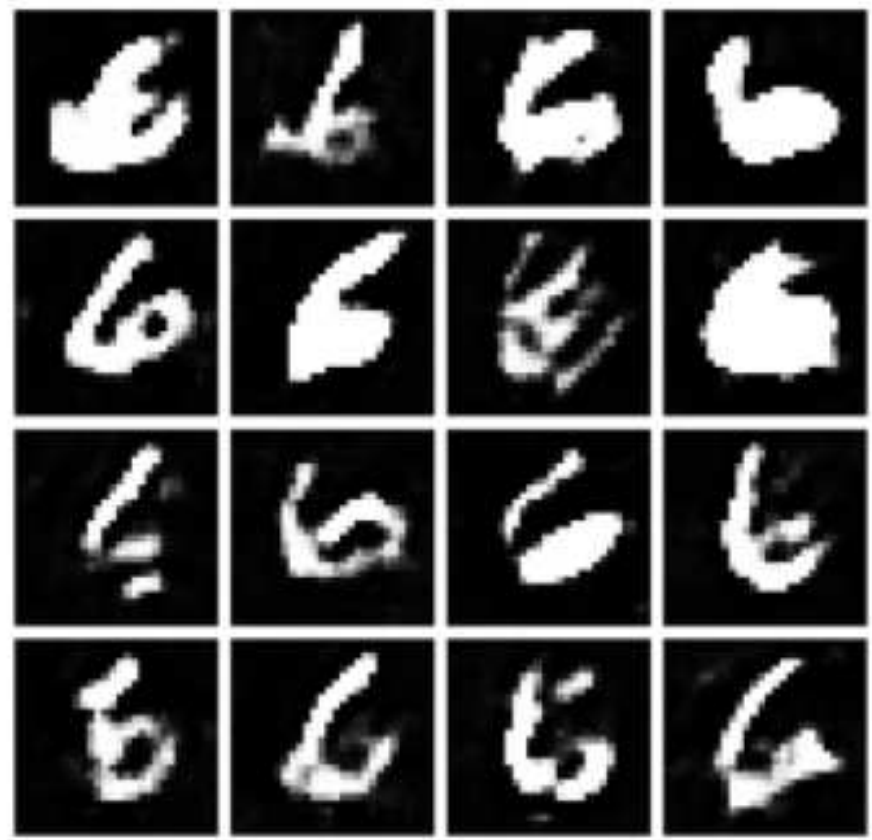

(b) Training 1000 times
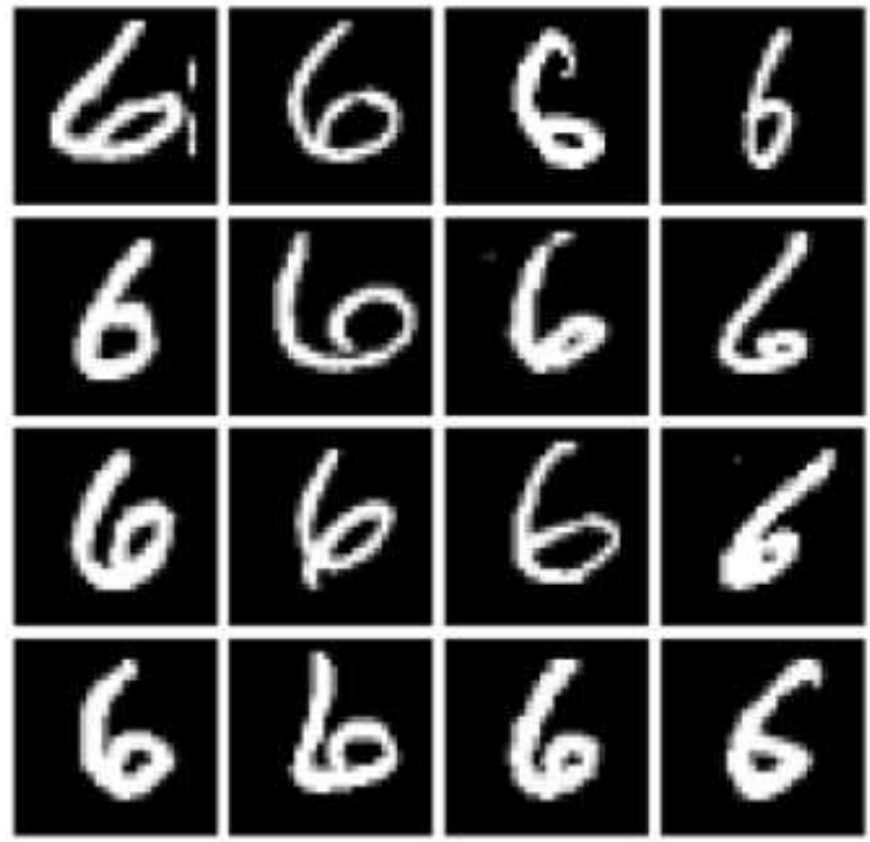

(d) Training 10000 times

Figure 5

Partial results of the DCGAN (Experiment 2) 


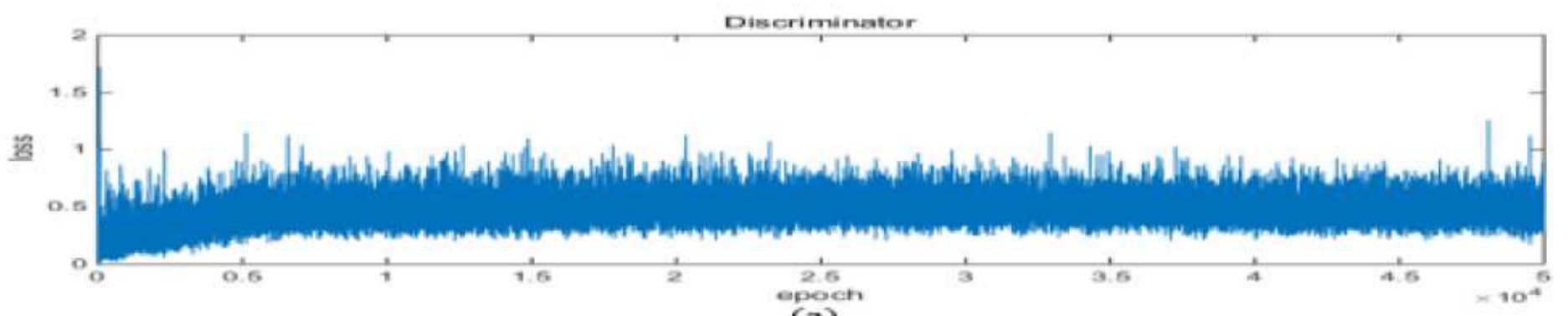

(a)

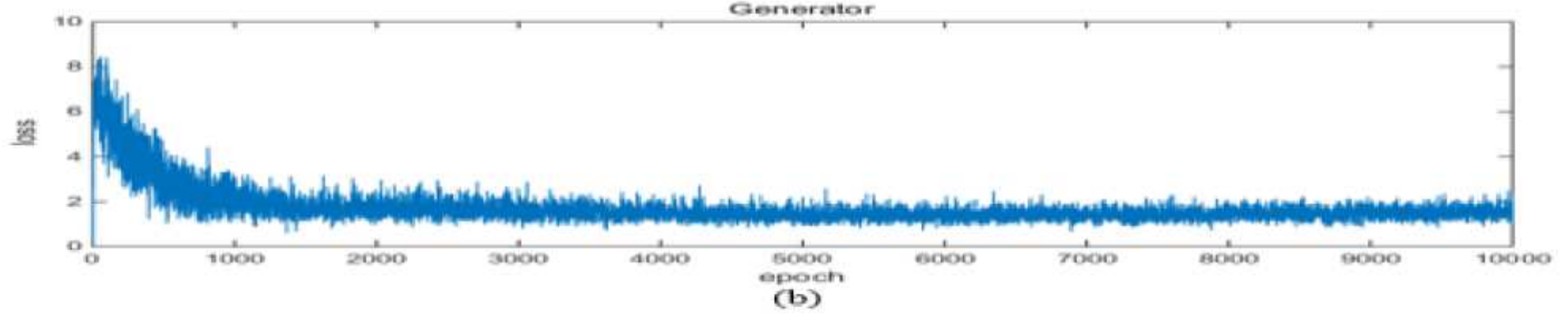

Figure 6

Loss curves of the DCGAN discriminator (a) and generator (b) (Experiment 2) 


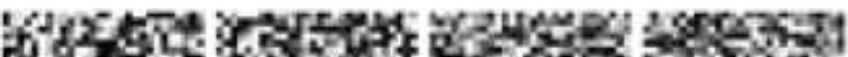

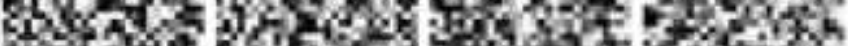

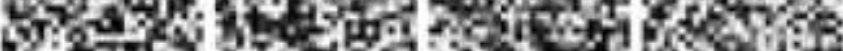

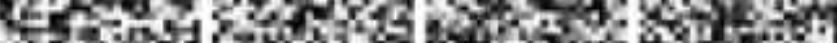
CRA

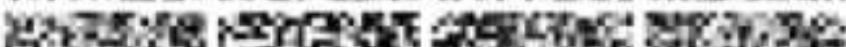
Tos

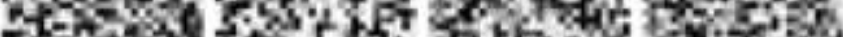

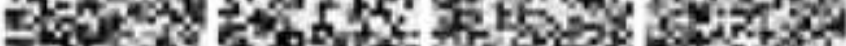

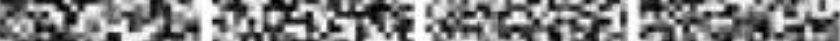

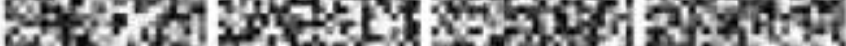

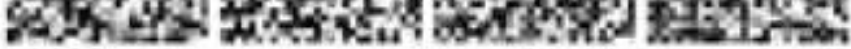

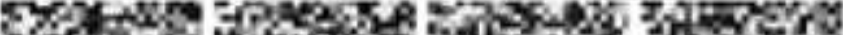

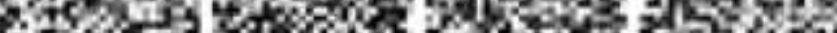

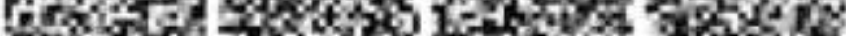

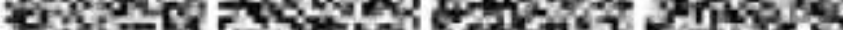

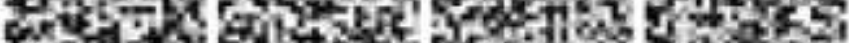

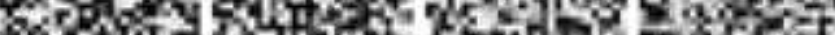

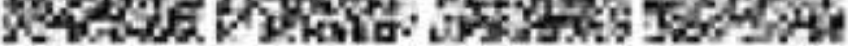

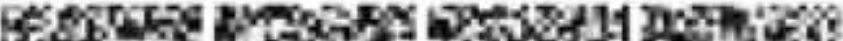

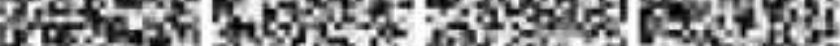

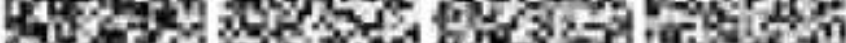

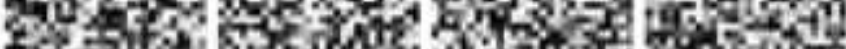

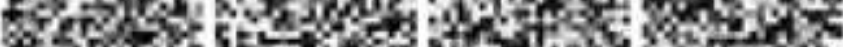

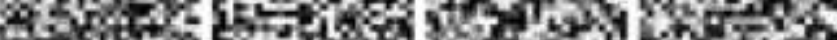

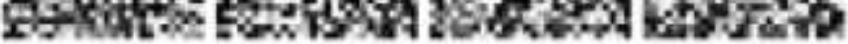

(a) Training 0 times
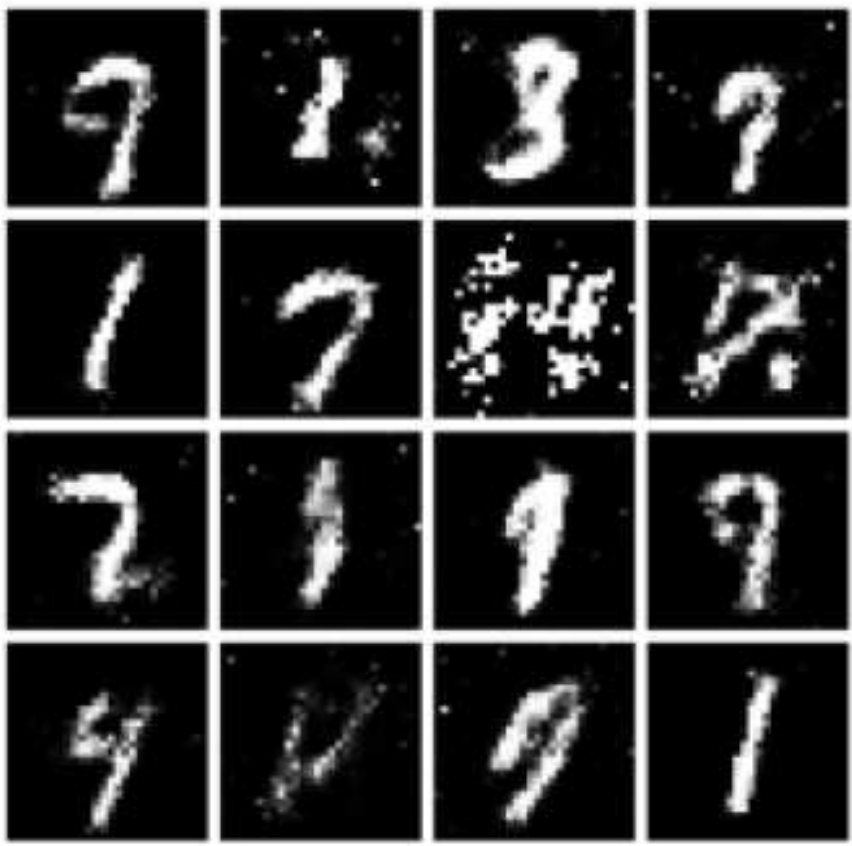

(c) Training 50000 times
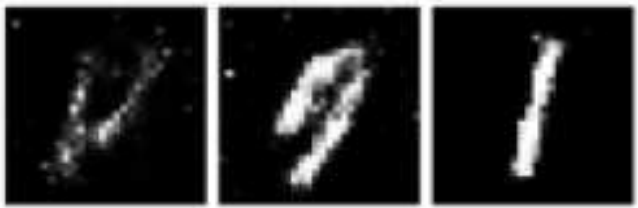
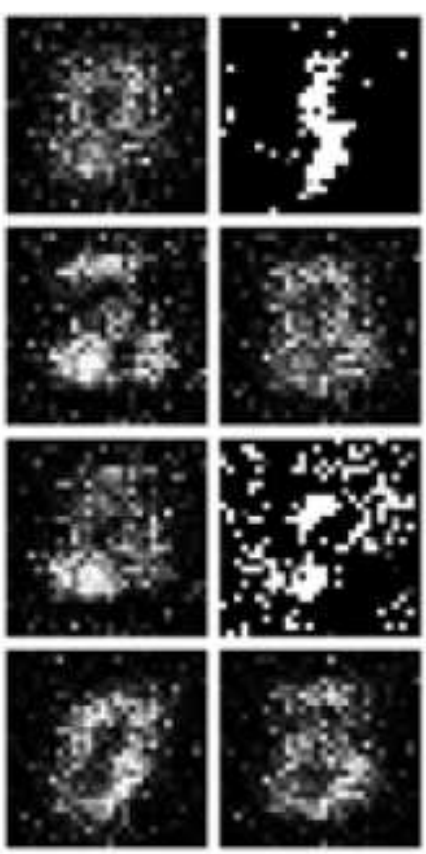

(b) Trai
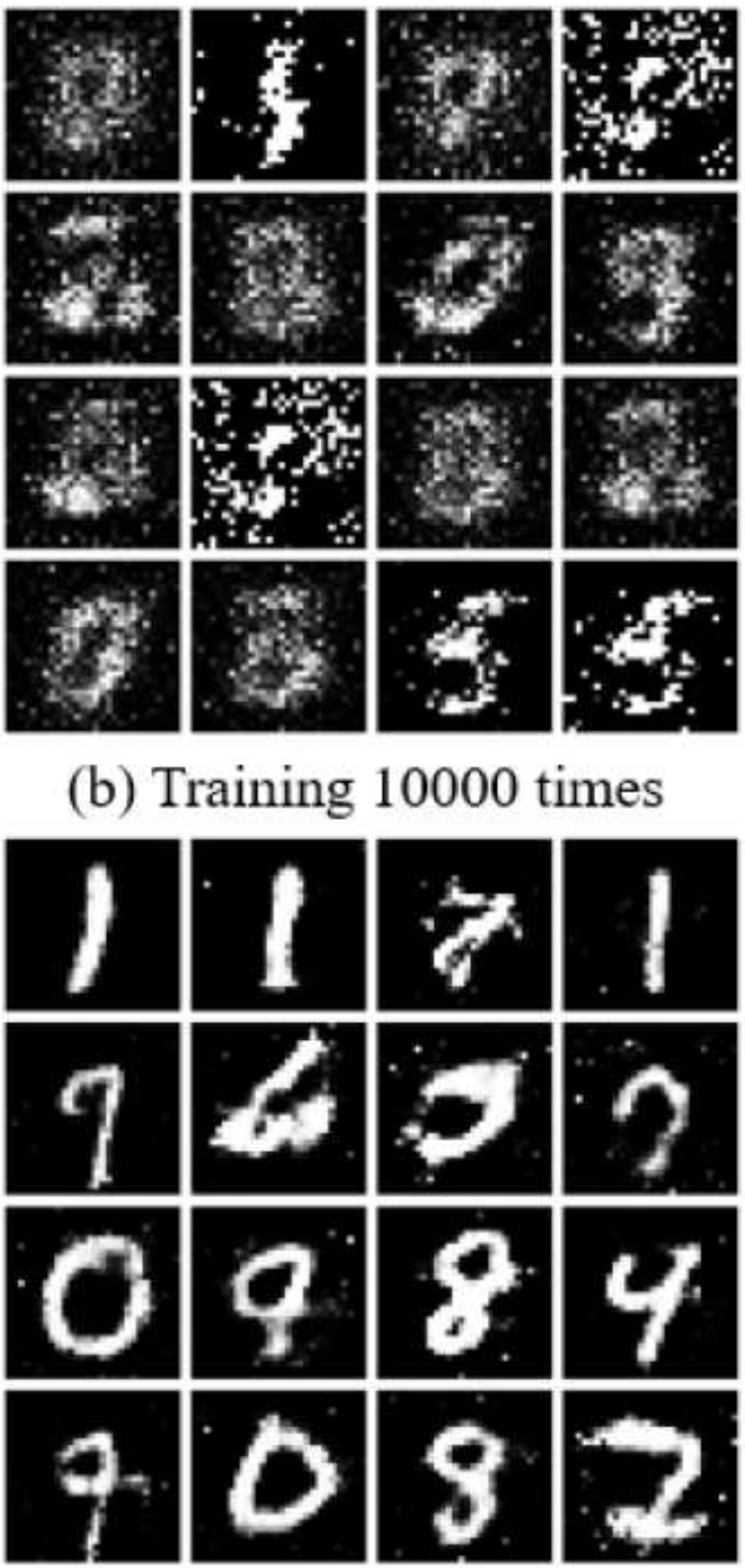

raining 10000 times
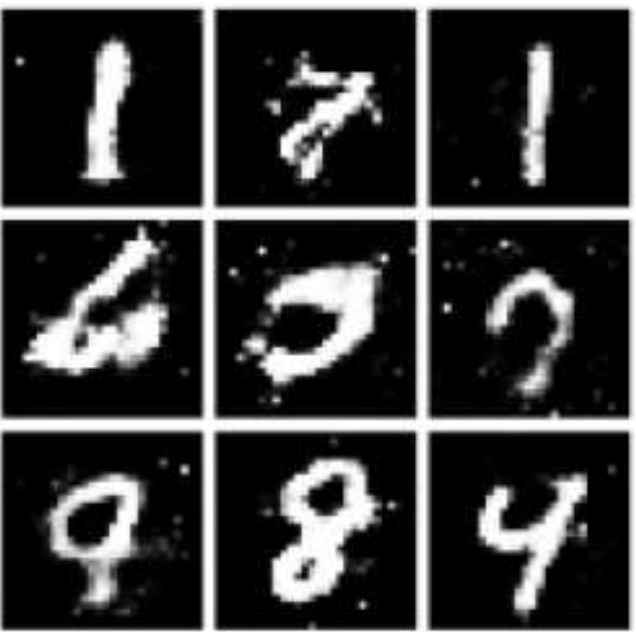

(d) Training 100000 times

Figure 7

Partial results of the GANs (Experiment 1) 
(a)

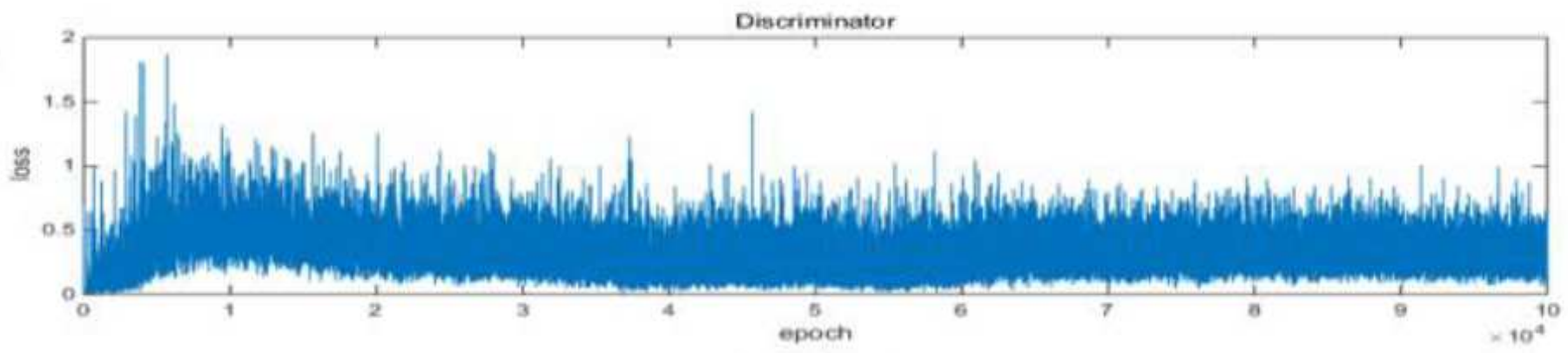

(b)

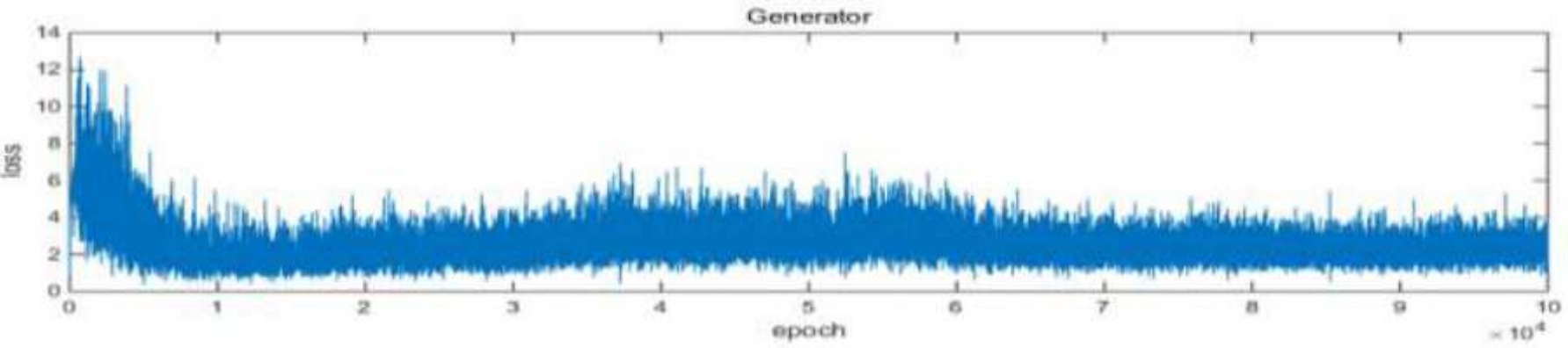

\section{Figure 8}

Loss curves of the GANs discriminator (a) and generator (b) (Experiment 1) 


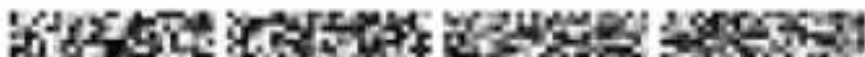

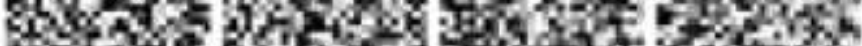

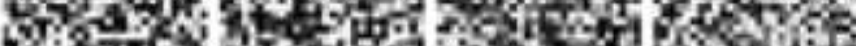

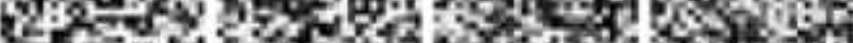

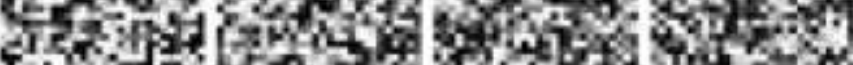

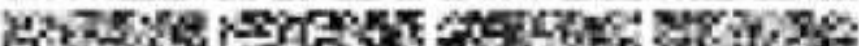

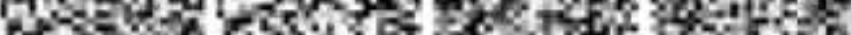

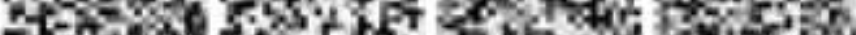

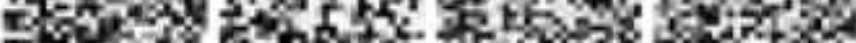

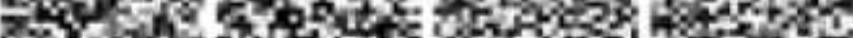

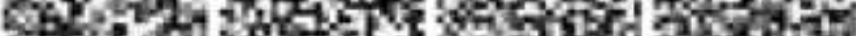

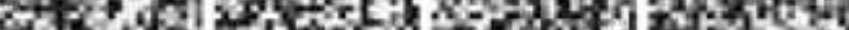

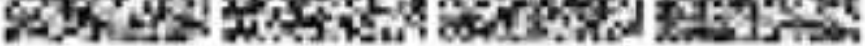

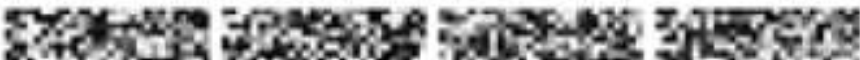

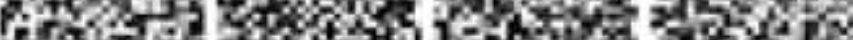

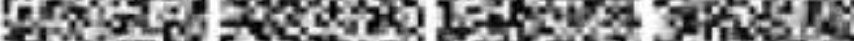

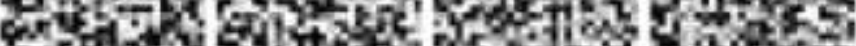

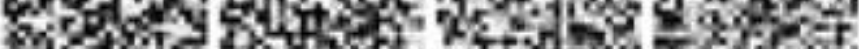

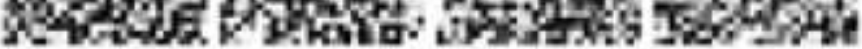

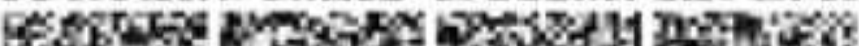

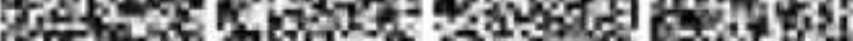

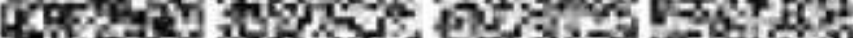

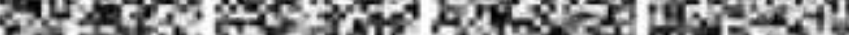

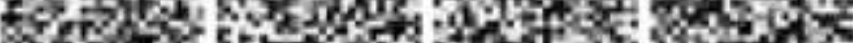

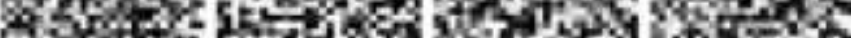

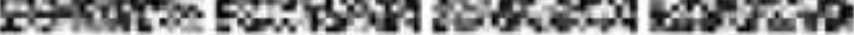

(a) Training 0 times
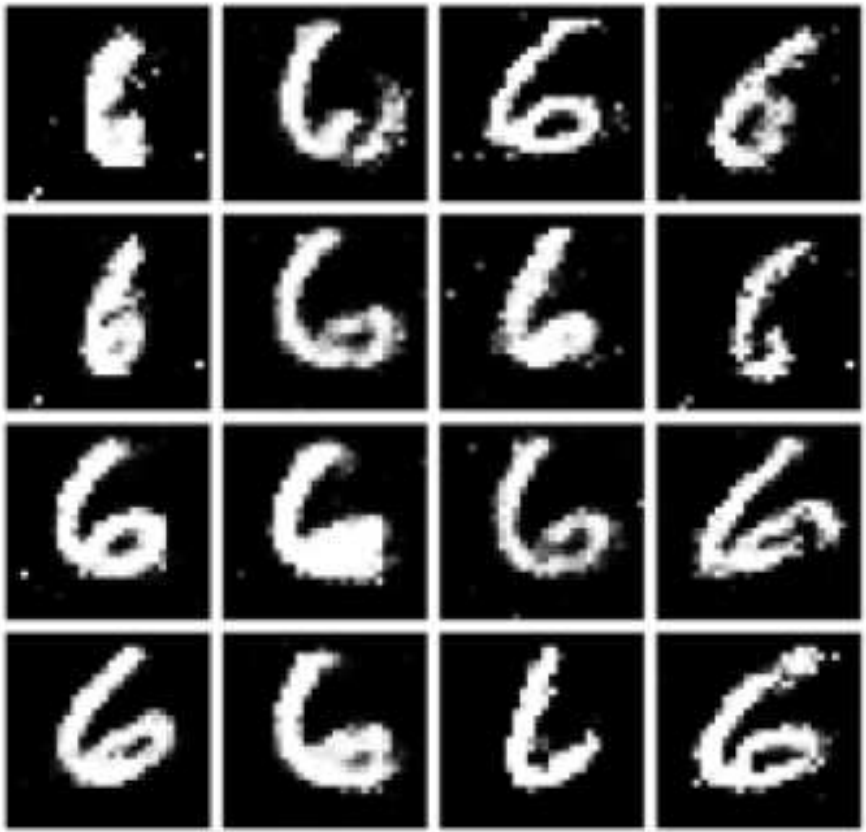

(c) Training 50000 times
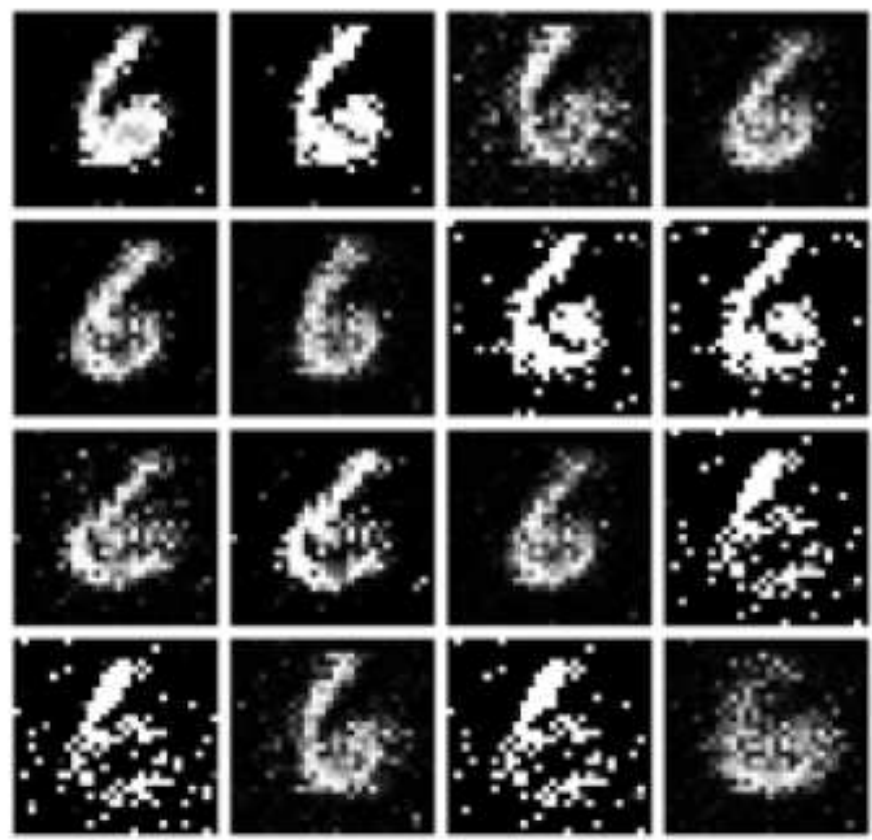

(b) Training 10000 times
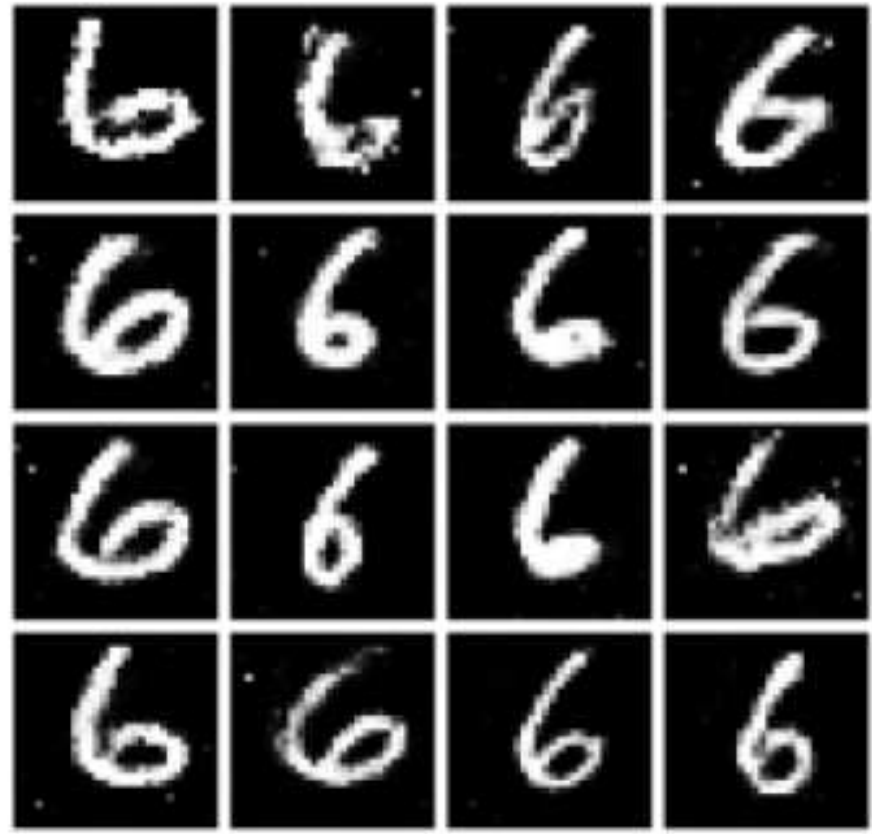

(d) Training 100000 times

Figure 9

Partial results of the GANs (Experiment 2) 

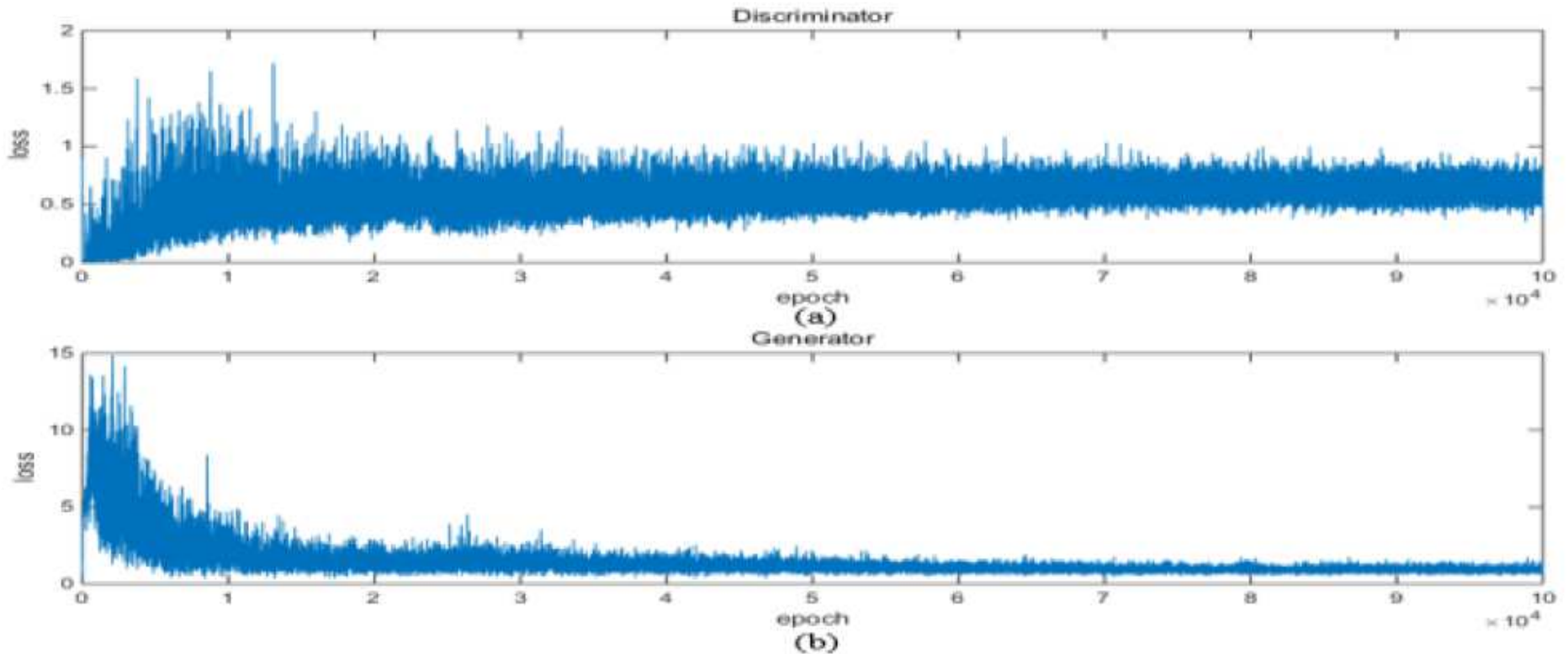

Figure 10

Loss curves of the GANs discriminator (a) and generator (b) (Experiment 2) 